\title{
Holomorphic functions, relativistic sum, Blaschke products and superoscillations
}

\author{
Daniel Alpay ${ }^{1} \cdot$ Fabrizio Colombo ${ }^{2} \cdot$ Stefano Pinton ${ }^{2} \cdot$ Irene Sabadini $^{2}$
}

Received: 24 October 2020 / Revised: 2 May 2021 / Accepted: 22 June 2021

/ Published online: 10 July 2021

(C) The Author(s) 2021

\begin{abstract}
Superoscillating functions are band-limited functions that can oscillate faster than their fastest Fourier component. The notion of superoscillation is a particular case of that one of supershift. In the recent years, superoscillating functions, that appear for example in weak values in quantum mechanics, have become an interesting and independent field of research in complex analysis and in the theory of infinite order differential operators. The aim of this paper is to study some infinite order differential operators acting on entire functions which naturally arise in the study of superoscillating functions. Such operators are of particular interest because they are associated with the relativistic sum of the velocities and with the Blaschke products. To show that some sequences of functions preserve the superoscillatory behavior it is of crucial importance to prove that their associated infinite order differential operators act continuously on some spaces of entire functions with growth conditions.
\end{abstract}

\section{Introduction}

Superoscillating function, or sequences, appear in weak values in quantum mechanics as shown in $[1,15]$ and in several other fields like optics or signal processing. Y.

$凶 \quad$ Irene Sabadini

irene.sabadini@polimi.it

Daniel Alpay

alpay@chapman.edu

Fabrizio Colombo

fabrizio.colombo@polimi.it

Stefano Pinton

stefano.pinton@polimi.it

1 Faculty of Mathematics, Physics, and Computation, Schmid College of Science and Technology, Chapman University, One University Drive, Orange, California 92866, USA

2 Politecnico di Milano Dipartimento di Matematica, Via E. Bonardi, 9, 20133 Milano, Italy

Birkhäuser 
Aharonov has suggested several problems associated with superoscillations and the two most important ones are: to establish how large is the class of superoscillatory functions and to study the evolution of superoscillations (associated with weak-values) as initial datum of Schrödinger equation or as initial condition of some relativistic quantum field equations.

The aim of this paper is to contribute to the first problem mentioned above. In fact, we show that one can construct superoscillatory functions or, more in general, functions that admit the supershift property by considering various tools such as the relativistic sum of the velocities and/or the Blaschke products in a way that will be specified in the sequel.

In the last decade a systematic study of superoscillating functions has been carried out from the mathematical point of view and extended to the case of several variables [10]. It has been shown that superoscillations can approximate functions in the Schwartz space $\mathcal{S}$, in the space of distributions of type $\mathcal{S}^{\prime}$, see [27], and of hyperfunctions as shown in [30]. Moreover, these functions appear in the Talbot effect, see [29], and supershifts for families of generalized functions are considered in [28]. We point out that the concept of supershift is more general than the one of superoscillation, which is a particular case. Finally, we mention that superoscillations are also useful in Schur analysis as shown in [16].

The rigorous treatment of the evolution of superoscillations, as initial datum of the Schrödinger equation, required some sophisticated mathematical tools, as it has been shown for example in [2-4,6-8,18,24-26] and in [11]. We also mention that evolution of superoscillations in discontinuous potentials is considered in $[3,9,19]$, while the evolution of superoscillations for Klein-Gordon and Dirac equations have been studied in [12] and [32], respectively.

The most important superoscillatory function, that appears in connection with weak values in quantum mechanics, is

$$
F_{n}(x, a)=\left(\cos \left(\frac{x}{n}\right)+i a \sin \left(\frac{x}{n}\right)\right)^{n}=\sum_{j=0}^{n} C_{j}(n, a) e^{i(1-2 j / n) x}, \quad x \in \mathbb{R},
$$

where $a>1$ and the coefficients $C_{j}(n, a)$ are given by

$$
C_{j}(n, a)=\left(\begin{array}{l}
n \\
j
\end{array}\right)\left(\frac{1+a}{2}\right)^{n-j}\left(\frac{1-a}{2}\right)^{j}, \text { for } j=0, \ldots, n \text { and } n \in \mathbb{N} \text {. }
$$

If we fix $x \in \mathbb{R}$ and we let $n$ go to infinity, we obtain that $\lim _{n \rightarrow \infty} F_{n}(x, a)=e^{i a x}$.

Inspired by (1) we define a more general class of superoscillatory functions.

We call generalized Fourier sequence a sequence of the form

$$
Y_{n}(x, a):=\sum_{j=0}^{n} E_{j}(n, a) e^{i k_{j}(n) x}, \quad x \in \mathbb{R},
$$

where $a \in \mathbb{R}, k_{j}(n) \in \mathbb{R}$ and $E_{j}(n, a) \in \mathbb{C}$ for all $j=0, \ldots, n$ and $n \in \mathbb{N}$. 
A generalized Fourier sequence $Y_{n}(x, a)$ is said to be a superoscillating sequence if it satisfy the two properties:

(P1) $\sup _{j, n}\left|k_{j}(n)\right| \leq 1$ for all $j=0, \ldots, n$ and $n \in \mathbb{N}$.

(P2) there exists a compact subset of $\mathbb{R}$, which will be called a superoscillation set, on which $\lim _{n \rightarrow \infty} Y_{n}(x, a)=e^{i g(a) x}$ where $g$ is a continuous function on an interval that contains the points $a, k_{j}(n)$ and such that $|g(a)|>1$.

As we have already mentioned superoscillations occur as a particular case of sequences of functions that satisfy the so called supershift property. This property is of crucial importance in the study of evolution of superoscillations as initial datum of the Schrödinger equation or of any other field equation in quantum mechanics and is defined below.

Supershift property. Let $\lambda \rightarrow \varphi_{\lambda}(x) \in \mathbb{C}$ be a continuous function in the variables $\lambda \in \mathcal{I}$, where $\mathcal{I} \subseteq \mathbb{R}$ is an interval, and $x \in \Omega$, where $\Omega$ is a domain. We consider $x \in \Omega$ as parameter for the function $\lambda \rightarrow \varphi_{\lambda}(x)$ where $\lambda \in \mathcal{I}$. When $[-1,1]$ is contained in $\mathcal{I}$ and $a \in \mathcal{I}$, we define the sequence

$$
\psi_{n}(t, x)=\sum_{j=0}^{n} C_{j}(n, a) \varphi_{1-2 j / n}(x)
$$

in which $\varphi_{\lambda}$ is computed just on the points $1-2 j / n$ belonging to the interval $[-1,1]$ and the coefficients $C_{j}(n, a)$ are defined for example as in (2), for $j=0, \ldots, n$ and $n \in \mathbb{N}$. If

$$
\lim _{n \rightarrow \infty} \psi_{n}(t, x)=\varphi_{a}(x)
$$

for $|a|>1$ arbitrary large (but belonging to $\mathcal{I}$ ), we say that the function $\lambda \rightarrow \varphi_{\lambda}(x)$, for $x$ fixed, admits a supershift in $\lambda$.

If we set $\varphi_{\lambda}(x)=e^{i \lambda x}$, we obtain the superoscillating sequence described above as a particular case of the supershift. In fact, in this case, we have $\psi_{n}(x)=F_{n}(x, a)$, where $F_{n}(x, a)$ is defined in (1). The name supershift is due to the fact that we are able to obtain $\varphi_{a}$, for $|a|>1$ arbitrarily large, by simply calculating the function $\lambda \rightarrow \varphi_{\lambda}$ in infinitely many points in the neighborhood $[-1,1]$ of the origin.

With the above definitions in mind we can now state our main results. In Sect. 2 we consider the two superoscillating functions (of supershifts):

$$
Y_{n}(x, a):=\sum_{j=0}^{n} C_{j}(n, a) e^{i \ell_{j}(n) x}, \quad Z_{n}(x, a):=\sum_{j=0}^{n} C_{j}(n, a) e^{i k_{j}(n) x}, \quad x \in \mathbb{R}
$$

and we define the pointwise product (related to the coefficients $\ell_{j}(n)$ and $k_{j}(n)$ ), denoted by ${ }_{P}$, as

$$
\left(Y_{n} \circ{ }_{P} Z_{n}\right)(x, a):=\sum_{j=0}^{n} C_{j}(n, a) e^{i \ell_{j}(n) k_{j}(n) x}, \quad x \in \mathbb{R} .
$$


In particular, we can set $\ell_{j}(n):=g(1-2 j / n)$ and $k_{j}(n):=h(1-2 j / n)$ where $g$ and $h$ are entire holomorphic functions. Moreover, we assume that $g$ and $h$ belong to the space of entire functions for which there exist constants $A, B>0$ such that $|g(z)| \leq A e^{B|z|^{p}}$ and $|h(z)| \leq A e^{B|z|^{p}}$ for some $p \in[1, \infty)$. In Theorem 2.8 we show the supershift property

$$
\lim _{n \rightarrow \infty}\left(Y_{n} \circ P Z_{n}\right)(x, a)=e^{i g(a) h(a) x}, \quad x \in \mathbb{R} \text { when }|a|>1
$$

as a consequence of the continuity of an infinite order differential operator associated with the function $Y_{n} \circ_{P} Z_{n}(x, a)$, see Theorem 2.7. When $g$ and $h$ are such that $g([-1,1]) \subseteq \mathbb{R}, h([-1,1]) \subseteq \mathbb{R}$, with the assumption that for $a \in \mathbb{R}$ we have $|g(a) h(a)|<1$ for $|a| \leq 1$ and $|g(a) h(a)|>1$ for $|a|>1$, then the sequence $F_{n} \circ{ }_{P} Y_{n}(x, a)$ is superoscillating. In the other cases we have the supershift property. To obtain superoscillations, a simple example of choice of $f$ and $g$ is given by $g(z)=z^{p}$ and $h(z)=z^{q}$ for $p, q \in \mathbb{N}$.

The relativistic sum of the velocities in special relativity suggests how to define a class of functions that admit the supershift property. We recall that in special relativity the law of addition of the velocities $v$ and $w$ of two objects moving along a line is given by

$$
u \oplus_{R} v=\frac{u+v}{1+u v / c^{2}}
$$

where $c$ is the speed of the light in the vacuum. When we normalize the speed of the light setting $c=1$ we have that if $|u|<1$ and $|v|<1$ then $\left|u \oplus_{R} v\right|<1$, see for more details [35]. This fact suggests how to define a binary operation between two sequences bounded by 1 that gives a sequence still bounded by 1 . Thus, in Sect. 3 we consider two superoscillating functions $Y_{n}(x, a), Z_{n}(x, a)$ and we define their relativistic product as:

$$
\left(Y_{n} \circ_{R} Z_{n}\right)(x, a):=\sum_{j=0}^{n} C_{j}(n, a) e^{i\left(\ell_{j}(n) \oplus_{R} k_{j}(n)\right) x}, \quad x \in \mathbb{R} .
$$

Here we assume that $\ell_{j}(n)$ and $k_{j}(n)$ are given by $\ell_{j}(n)=g(\varepsilon(1-2 j / n))$ and $k_{j}(n)=h(\varepsilon(1-2 j / n))$, for $\varepsilon \in(0,1)$, where $g$ and $h$ are holomorphic functions defined on the unit ball $\mathbb{D}$ in $\mathbb{C}$ that satisfy suitable conditions. Then we prove that

$$
\lim _{n \rightarrow \infty}\left(Y_{n} \circ_{R} Z_{n}\right)(x, a)=e^{i x\left(g(\varepsilon a) \oplus_{R} h(\varepsilon a)\right)}, \quad x \in \mathbb{R},
$$

for any $\varepsilon a<1$ using suitable defined infinite order differential operators that depend on the relativistic sum.

The technique used to prove our main results is based on infinite order differential operators $\mathcal{U}\left(x, \partial_{\xi}\right)$ that are applied to the fundamental superoscillating function (1). We then define

$$
\psi_{n}(x):=\left.\mathcal{U}\left(x, \partial_{\xi}\right) F_{n}(\xi, a)\right|_{\xi=0}
$$


where $\left.\right|_{\xi=0}$ denotes the restriction to $\xi=0$ with respect to the auxiliary complex variable $\xi$. A crucial fact in the strategy of generating superoscillating functions is the study of the continuity of the operator $\mathcal{U}\left(x, \partial_{\xi}\right)$ on the space of entire functions with growth conditions. As we will see in Sect. 3 the infinite order differential operator associated with the relativistic sum is of the form

$$
\mathcal{U}_{R}\left(x, \partial_{\xi}\right)=\sum_{m=0}^{\infty} \frac{(i x)^{m}}{m !} C^{m}\left(\partial_{\xi}\right)
$$

where

$C\left(\partial_{\xi}\right)=B\left(\partial_{\xi}\right) \sum_{\ell=0}^{\infty}(-1)^{\ell} A^{\ell}\left(\partial_{\xi}\right), \quad A\left(\partial_{\xi}\right)=\sum_{p=0}^{\infty} a_{p} i^{-p} \partial_{\xi}^{p}, \quad B\left(\partial_{\xi}\right)=\sum_{q=0}^{\infty} b_{q} i^{-q} \partial_{\xi}^{q}$

and the coefficients of the $\left(a_{p}\right)_{p \in \mathbb{N}_{0}}$ and $\left(b_{p}\right)_{p \in \mathbb{N}_{0}}$ in the formal operators $A\left(\partial_{\xi}\right)$ and $B\left(\partial_{\xi}\right)$ acting on entire functions are sequences of complex numbers that satisfy suitable growth conditions, see Theorem 3.9. In Sect. 4 we study the infinite order differential operators associated with the Blaschke products and we use such operators to study superoscillatory functions.

Blaschke products provide another case that we study in order to get sequences that admit the supershift property. The corresponding infinite order differential operators are studied in Sect. 4.

Remark 1.1 To determine the structure of the infinite order differential operators associated with the relativistic sum and the Blaschke product is part of our task. The precise identification of these infinite order differential operators is useful also for further investigations. For example, the study of the supershift property in the space of hyperfunctions has been done for the infinite order differential operator associated with the quantum harmonic oscillator, see [31].

We conclude this introduction, by pointing out that a different class of superoscillating functions with respect to the ones considered in this paper can be found in [23], and a very general technique to obtain superoscillations has been recently introduced in the paper [5] and explained in the last section.

\section{Superoscillations and supershift}

We recall some definitions and results on entire functions, see e.g. [20], which will play an important role in the proofs of the main results.

Definition 2.1 Let $p \geq 1$. We denote by $\mathcal{A}_{p}$ the space of entire functions with order lower than $p$ also called the space of entire functions of order $p$ and finite type. It consists of functions $f$ for which there exist constants $B, C>0$ such that

$$
|f(z)| \leq C e^{B|z|^{p}} .
$$


Let $\left(f_{n}\right)_{n \in \mathbb{N}}, f_{0} \in \mathcal{A}_{p}$. Then $f_{n} \rightarrow f_{0}$ in $\mathcal{A}_{p}$ if there exists some $B>0$ such that

$$
\lim _{n \rightarrow \infty} \sup _{z \in \mathbb{C}}\left|\left(f_{n}(z)-f_{0}(z)\right) e^{-B|z|^{p}}\right|=0
$$

The following result gives a characterization of functions in $\mathcal{A}_{p}$ in terms of their Taylor coefficients.

Lemma 2.2 (Lemma 2.2 in [18]) Let $p \geq 1$. A function $f(z)=\sum_{j=0}^{\infty} f_{j} z^{j}$ belongs to $\mathcal{A}_{p}$, i.e., there exist positive constants $B, C>0$ such that $|f(z)| \leq C e^{B|z|^{p}}$, if and only if there exist constants $C_{f}, b_{f}>0$ such that

$$
\left|f_{j}\right| \leq C_{f} \frac{b_{f}^{j}}{\Gamma\left(\frac{j}{p}+1\right)},
$$

where the constant $b_{f}$ is given by

$$
b_{f}=\left(2^{p} B p e\right)^{1 / p} \text {. }
$$

Furthermore, a sequence $f_{n}$ in $\mathcal{A}_{p}$ tends to zero if and only if $C_{f_{n}} \rightarrow 0$ and $b_{f_{n}}<b$ for some $b>0$.

Remark 2.3 The constant $C>0$ in the estimate (6), i.e., $|f(z)| \leq C e^{B|z|^{p}}$, and the constant $C_{f}>0$ in estimate (8), i.e., $\left|f_{j}\right| \leq C_{f} \frac{b_{f}^{j}}{\Gamma\left(\frac{j}{p}+1\right)}$, are in general different. From an inspection of the proof of Lemma 2.2 in [18] the relation between the constants $B>0$ and $b_{f}>0$ is given by (9), i.e., $b_{f}=\left(2^{p} B p e\right)^{1 / p}$, where $e$ is the Neper number.

The holomorphic extension of $F_{n}(x, a)$ as in (1) converges in $\mathcal{A}_{1}$ as the following lemma, proved in [28], shows.

Lemma 2.4 Let $a \in \mathbb{C}$, for any $z \in \mathbb{C}$, consider

$$
F_{n}(z, a):=\left(\cos \left(\frac{z}{n}\right)+i a \sin \left(\frac{z}{n}\right)\right)^{n}
$$

with $z, a \in \mathbb{C}$. Then, for every $n \in \mathbb{N}$ and $z \in \mathbb{C}$, one has

$$
\left|F_{n}(z, a)\right| \leq \exp (|a||z|+|\operatorname{Im}(z)|) \leq \exp ((|a|+1)|z|)
$$

Now we will consider generalized Fourier sequences $\left(\sum_{j=0}^{n} C_{j}(n, a) e^{i k_{j}(n) x}\right)_{n}$ in which the exponential sequences $\left(k_{j}(n)\right)_{j, n}$ are defined by holomorphic functions in $\mathcal{A}_{q}$ for some $q \geq 1$. 
Definition 2.5 (Generalized Fourier sequences depending on an entire function) Let $a \in \mathbb{R}$ and $C_{j}(n, a) \in \mathbb{C}$ be as in (2) for all $j=0, \ldots, n$ and $n \in \mathbb{N}$. Let $h \in \mathcal{A}_{q}$, for some $q \geq 1$, be such that $h$ maps $\mathbb{R}$ to itself, we define the generalized Fourier series

$$
Y_{n}(x, a):=\sum_{j=0}^{n} C_{j}(n, a) e^{i k_{j}(n) x},
$$

where $k_{j}(n):=h(1-2 j / n)$ for all $j=0, \ldots, n$ and $n \in \mathbb{N}$.

Definition 2.6 (The product $\circ_{P}$ of generalized Fourier sequences) Let $Y_{n}(x, a)$ and $Z_{n}(x, a)$ be generalized Fourier sequences as in Definition 2.5, i.e.,

$$
Y_{n}(x, a):=\sum_{j=0}^{n} C_{j}(n, a) e^{i \ell_{j}(n) x}, \quad Z_{n}(x, a):=\sum_{j=0}^{n} C_{j}(n, a) e^{i k_{j}(n) x}, \quad x \in \mathbb{R}
$$

where $C_{j}(n, a) \in \mathbb{C}$ be as in (2) for all $j=0, \ldots, n$ and $n \in \mathbb{N}$. Let $h, g \in \mathcal{A}_{q}$, for some $q \geq 1$, be such that $h$ and $g$ map $\mathbb{R}$ to itself, $\ell_{j}(n):=h(1-2 j / n)$ and $k_{j}(n):=g(1-2 j / n)$. We define the product $o_{P}$ of the sequences $Y_{n}(x, a)$ and $Z_{n}(x, a)$ by

$$
\left(Y_{n} \circ P Z_{n}\right)(x, a):=\sum_{j=0}^{n} C_{j}(n, a) e^{i \ell_{j}(n) k_{j}(n) x}, \quad x \in \mathbb{R} .
$$

In order to compute the $\operatorname{limit}_{n \rightarrow \infty} \lim _{n} \circ_{P} Z_{n}(x, a)$ we need to define a suitable infinite order differential operator acting on $\mathcal{A}_{1}$ and to prove the continuity of such operator on $\mathcal{A}_{1}$. Thanks to the assumptions on the functions $h, g \in \mathcal{A}_{q}$, for some $q \geq 1$, we will prove the supershift property for function $Y_{n} \circ_{P} Z_{n}(x, a)$ and if $h$ and $g$ satisfy additional conditions, then the sequence $Y_{n} \circ_{P} Z_{n}(x, a)$ is, in particular, superoscillating.

Theorem 2.7 Assume that $x$ is a fixed real parameter and let $\xi \in \mathbb{C}$. We define the formal infinite order differential operator:

$$
\mathcal{U}\left(x, \partial_{\xi}\right):=\sum_{m=0}^{\infty} \frac{(i x)^{m}}{m !} A^{m}\left(\partial_{\xi}\right): \mathcal{A}_{1} \rightarrow \mathcal{A}_{1}, \quad x \in \mathbb{R}
$$

where the operator $A\left(\partial_{\xi}\right)$ is defined replacing $z$ by $i^{-1} \partial_{\xi}$ in the entire function

$$
G(z)=\sum_{p=0}^{\infty} a_{p} z^{p} \in \mathcal{A}_{q}
$$


i.e.,

$$
A\left(\partial_{\xi}\right):=\sum_{p=0}^{\infty} a_{p} i^{-p} \partial_{\xi}^{p} .
$$

Then $\mathcal{U}\left(x, \partial_{\xi}\right): \mathcal{A}_{1} \rightarrow \mathcal{A}_{1}$ is a continuous linear operator. Moreover, we have

$$
\left.\left(\mathcal{U}\left(x, \partial_{\xi}\right) e^{i a \xi}\right)\right|_{\xi=0}=e^{i G(a) x} .
$$

Proof First we consider the action of the operators $A^{m}\left(\partial_{\xi}\right), m \in \mathbb{N}$, on functions $f \in \mathcal{A}_{1}, f(\xi)=\sum_{j=0}^{\infty} f_{j} \xi^{j}, f_{j} \in \mathbb{C}$. We claim that

$$
\begin{aligned}
A^{m}\left(\partial_{\xi}\right)(f)= & \sum_{p_{1}=0, \ldots, p_{m}=0}^{\infty} a_{p_{1}} \cdots a_{p_{m}} i^{-\left(p_{1}+\cdots+p_{m}\right)} \\
& \times \sum_{j=0}^{\infty} f_{p_{1}+\cdots+p_{m}+j} \frac{\left(p_{1}+\cdots+p_{m}+j\right) !}{j !} \xi^{j} .
\end{aligned}
$$

The proof of the above formula is by induction. First we observe that for $m=1$ we have

$$
\begin{aligned}
A\left(\partial_{\xi}\right)(f) & =\sum_{p=0}^{\infty} a_{p} i^{-p} \partial_{\xi}^{p}(f(\xi)) \\
& =\sum_{p=0}^{\infty} a_{p} i^{-p} \partial_{\xi}^{p}\left(\sum_{j=0}^{\infty} f_{j} \xi^{j}\right) \\
& =\sum_{p=0}^{\infty} a_{p} i^{-p} \sum_{j=p}^{\infty} f_{j} \xi^{j-p} \frac{j !}{(j-p) !}=\sum_{p=0}^{\infty} a_{p} i^{-p} \sum_{j=0}^{\infty} f_{j+p} \xi^{j} \frac{(j+p) !}{j !}
\end{aligned}
$$

Thus the formula (12) is true for $m=1$. If we suppose that the formula (12) holds for $m=n$ then for $m=n+1$ we have

$$
\begin{aligned}
A^{n+1}\left(\partial_{\xi}\right)(f)= & A^{n}\left(\partial_{\xi}\right)\left(A\left(\partial_{\xi}\right)(f)\right)=A^{n}\left(\partial_{\xi}\right)\left(\sum_{p=0}^{\infty} \sum_{j=0}^{\infty} a_{p} i^{-p} f_{j+p} \frac{(j+p) !}{j !} \xi^{j}\right) \\
= & \sum_{p_{1}=0, \ldots, p_{n}=0}^{\infty} a_{p_{1}} \cdots a_{p_{n}} i^{-\left(p_{1}+\cdots+p_{n}\right)} \\
& \times \sum_{p=0}^{\infty} \sum_{k=0}^{\infty} a_{p} i^{-p} f_{p_{1}+\cdots+p_{n}+p+k} \frac{\left(p_{1}+\cdots+p_{n}+p+k\right) !}{\left(p_{1}+\cdots+p_{n}+k\right) !}
\end{aligned}
$$




$$
\begin{aligned}
& \times \frac{\left(p_{1}+\cdots+p_{n}+k\right) !}{k !} \xi^{k} \\
= & \sum_{p_{1}=0, \ldots, p_{n+1}=0}^{\infty} a_{p_{1}} \cdots a_{p_{n+1}} i^{-\left(p_{1}+\cdots+p_{n+1}\right)} \\
& \times \sum_{k=0}^{\infty} f_{p_{1}+\cdots+p_{n+1}+k} \frac{\left(p_{1}+\cdots+p_{n+1}+k\right) !}{k !} \xi^{k},
\end{aligned}
$$

where in the third equality we set $k=j-p_{1}-\cdots-p_{n}$ and in the last equality we rename the index $p$ by $p_{n+1}$. Thus the proof of the formula (12) is complete.

We recall that if $G \in \mathcal{A}_{q}$, for $q \geq 1$, by Lemma 2.2, there exist positive constants $b_{G}, C_{G}$ such that

$$
\left|a_{j}\right| \leq C_{G} \frac{b_{G}^{j}}{\Gamma\left(\frac{j}{q}+1\right)},
$$

and in particular if $f \in \mathcal{A}_{1}, f(z)=\sum_{j=0}^{\infty} f_{j} z^{j}$, then $\left|f_{j}\right| \leq C_{f} \frac{b_{f}^{j}}{j !}$ for some positive constants $b_{f}, C_{f}$. Using these estimates we have

$$
\begin{aligned}
\mid \mathcal{U} & \left(x, \partial_{\xi}\right) f(\xi)\left|\leq \sum_{m=0}^{\infty} \frac{|x|^{m}}{m !}\right| A^{m}\left(\partial_{\xi}\right) f(\xi) \mid \\
& \leq \sum_{m=0}^{\infty} \frac{|x|^{m}}{m !} \sum_{p_{1}=0, \ldots, p_{m}=0}^{\infty}\left|a_{p_{1}} \cdots a_{p_{m}}\right| \\
& \sum_{j=0}^{\infty} C_{f} \frac{b_{f}^{p_{1}+\cdots+p_{m}+j}}{\left(p_{1}+\cdots+p_{m}+j\right) !} \frac{\left(p_{1}+\cdots+p_{m}+j\right) !}{j !}|\xi|^{j} \\
& \leq C_{f} e^{b_{f}|\xi|} \sum_{m=0}^{\infty} \frac{|x|^{m}}{m !} C_{G}^{m} \sum_{p_{1}=0, \ldots, p_{m}=0}^{\infty} \frac{\left(b_{G} b_{f}\right) p_{1}+\cdots+p_{m}}{\Gamma(1) \cdots \Gamma\left(\frac{p_{m}}{q}+1\right)} \\
& \leq C_{f} e^{b_{f}|\xi|} \sum_{m=0}^{\infty} \frac{|x|^{m}}{m !} C_{G}^{m} E_{q}^{m}\left(b_{G} b_{f}\right) \\
& \leq C_{f} \exp \left(b_{f}|\xi|+C_{G} E_{q}\left(b_{G} b_{f}\right)|x|\right),
\end{aligned}
$$

where in the fourth inequality we have used that the series are absolute convergent and

$$
E_{q}(z):=\sum_{p=0}^{\infty} \frac{z^{p}}{\Gamma\left(\frac{p}{q}+1\right)}
$$


is the Mittag-Leffler function of order $q$. The previous inequality means that $\mathcal{U}\left(x, \partial_{\xi}\right) f(\xi) \in \mathcal{A}_{1}$ and also that $\mathcal{U}\left(x, \partial_{\xi}\right)$ is continuous over $\mathcal{A}_{1}$, i.e,. $\mathcal{U}\left(x, \partial_{\xi}\right) f_{n}(\xi) \rightarrow$ 0 as $f_{n} \rightarrow 0$. Observe that instead of $f_{n} \rightarrow 0$ we can equivalently require that $C_{f_{n}} \rightarrow 0$ with $b_{f_{n}} \leq b_{0}$, for some positive constant $b_{0}$, where the constants $C_{f_{n}}$ and $b_{f_{n}}$ refer to $\left|f_{n, j}\right| \leq C_{f_{n}} \frac{b_{f_{n}}^{j}}{j !}$. Moreover, we have that

$$
\begin{aligned}
\left.\left(\mathcal{U}\left(x, \partial_{\xi}\right) e^{i a \xi}\right)\right|_{\xi=0}= & \left(\sum_{m=0}^{\infty} \frac{(i x)^{m}}{m !} \sum_{p_{1}=0, \ldots, p_{m}=0}^{\infty} a_{p_{1}} \cdots a_{p_{m}} i^{-\left(p_{1}+\cdots+p_{m}\right)}\right. \\
& \left.\times \sum_{k=0}^{\infty} \frac{(i a)^{p_{1}+\cdots+p_{m}+k}\left(p_{1}+\cdots+p_{m}+k\right) !}{\left(p_{1}+\cdots+p_{m}+k\right) ! k !} \xi^{k}\right)\left.\right|_{\xi=0} \\
= & \sum_{m=0}^{\infty} \frac{(i x)^{m}}{m !}\left(\sum_{p_{1}=0}^{\infty} a_{p_{1}}(a)^{p_{1}}\right) \times \cdots \\
& \times\left(\sum_{p_{m}=0}^{\infty} a_{p_{m}}(a)^{p_{m}}\right)=\sum_{m=0}^{\infty} \frac{(i x)^{m}}{m !}(G(a))^{m}=e^{i G(a) x}
\end{aligned}
$$

where in the second equality we have used the absolute convergence for $a$ fixed.

Now we can prove the superoscillating property of the product of functions in Definition 2.6.

Theorem 2.8 Let $h, g \in \mathcal{A}_{q}$, for some $q \geq 1$, be such that $h$ and $g$ map $\mathbb{R}$ to itself. Let $Y_{n}(x, a)$ and $Z_{n}(x, a)$, for $x \in \mathbb{R}$ and $|a|>1$, be as in the Definition 2.6. Then we have

$$
\begin{gathered}
\lim _{n \rightarrow \infty}\left(Y_{n} \circ P\right. \\
\left.Z_{n}\right)(x, a)=\lim _{n \rightarrow \infty} \sum_{j=0}^{n} C_{j}(n, a) e^{i h(1-2 j / n) g(1-2 j / n) x} \\
=e^{i g(a) h(a) x}, \quad x \in \mathbb{R} .
\end{gathered}
$$

Moreover, if $|g(a) h(a)|<1$ for $|a| \leq 1$ and $|g(a) h(a)|>1$ for $|a|>1$ then the sequence $\left(Y_{n} \circ_{P} Z_{n}\right)(x, a)$ is superoscillating.

Remark 2.9 In Theorem 2.8, if we consider the supershift property instead of the superoscillating property, we do not have to require that the functions $g$ and $h$ to map $\mathbb{R}$ to itself.

Proof Let $h, g \in \mathcal{A}_{q}$ for some $q \geq 1$. We consider the function

$$
\left(Y_{n} \circ{ }_{P} Z_{n}\right)(x, a)=\sum_{j=0}^{n} C_{j}(n, a) e^{i h\left(1-2 \frac{j}{n}\right) g\left(1-2 \frac{j}{n}\right) x}, \quad a, x \in \mathbb{R} .
$$


Since $h, g \in \mathcal{A}_{q}$ the function $G(z):=h(z) g(z)$ belongs to $\mathcal{A}_{q}$. We define the operator $\mathcal{U}\left(x, \partial_{\xi}\right)$ as in Theorem 2.7 and thus we obtain the relations

$$
e^{i x G(1-2 j / n)}=\left.\mathcal{U}\left(x, \partial_{\xi}\right) e^{i \xi(1-2 j / n)}\right|_{\xi=0}
$$

and

$$
\left.\mathcal{U}\left(x, \partial_{\xi}\right) e^{i a \xi}\right|_{\xi=0}=e^{i x G(a)} .
$$

Thus we have

$$
\begin{aligned}
\lim _{n \rightarrow \infty}\left(Y_{n} \circ Z_{n}\right)(x, a) & =\lim _{n \rightarrow \infty} \sum_{j=0}^{n} C_{j}(n, a) e^{i x G(1-2 j / n)} \\
& \left.\stackrel{(13)}{=} \lim _{n \rightarrow \infty} \sum_{j=0}^{n} C_{j}(n, a) \mathcal{U}\left(x, \partial_{\xi}\right) e^{i \xi(1-2 j / n)}\right|_{\xi=0} \\
& =\left.\lim _{n \rightarrow \infty} \mathcal{U}\left(x, \partial_{\xi}\right) \sum_{j=0}^{n} C_{j}(n, a) e^{i \xi(\varepsilon(1-2 j / n))}\right|_{\xi=0}
\end{aligned}
$$

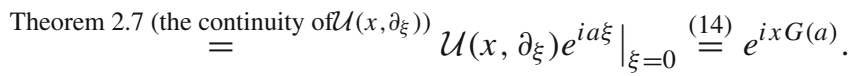

Remark 2.10 A simple example for the superoscillatory case is $g(z)=z^{l}$ and $h(z)=$ $z^{s}$ for $l, s \in \mathbb{N}$.

\section{The relativistic sum and superoscillating functions}

In the previous section we have considered generalized Fourier sequences as in Definition 2.6, where the product $o_{P}$ involves the pointwise product of the coefficients $\ell_{j}(n)$ and $k_{j}(n)$ of the exponentials in $Y_{n}(x, a)$ and $Z_{n}(x, a)$, respectively. Using the ${ }^{\circ} P$ product it is clear that, if we are in the superoscillating case where $\left|\ell_{j}(n)\right|<1$ and $\left|k_{j}(n)\right|<1$, then we have $\left|\ell_{j}(n) k_{j}(n)\right|<1$. But if we consider the sum $\ell_{j}(n)+k_{j}(n)$ instead of the product $\ell_{j}(n) k_{j}(n)$ we cannot guarantee that $\left|\ell_{j}(n)+k_{j}(n)\right|<1$. An interesting sum that overcomes this fact is the relativistic sum of velocities, see [35] where we can set $c=1$ so that

$$
u \oplus_{R} v=\frac{u+v}{1+u v} .
$$

We denote by $\mathbb{D}$ the open unit disc in $\mathbb{C}$. An important point in our considerations is the following result.

Lemma 3.1 Let $g, h: \mathbb{D} \rightarrow \mathbb{D}$ be two holomorphic functions.

(I) The relativistic sum of $g$ and $h$

$$
\left(g \oplus_{R} h\right)(z):=\frac{g(z)+h(z)}{1+g(z) h(z)}
$$


is a holomorphic function on $\mathbb{D}$.

(II) If $u, v \in(-1,1)$ then $\left|u \oplus_{R} v\right|<1$.

Proof Point (I) follows by observing that $|g(z) h(z)|<1$. Point (II) can be deduced from the observation that $u \oplus_{R} v$ is the restriction to the square $(-1,1) \times(-1,1)$ of the Möbius map

$$
\left(z_{1}, z_{2}\right) \rightarrow \frac{z_{1}+z_{2}}{1+\overline{z_{1}} z_{2}}
$$

that sends $\mathbb{D} \times \mathbb{D}$ to $\mathbb{D}$ where $z_{1}=u+i l$ and $z_{2}=v+i s$.

Remark 3.2 In general, the function $g \oplus_{R} h$ defined in the previous lemma, does not map $\mathbb{D}$ in $\mathbb{D}$. For example we can consider $h(z)=g(z) \equiv \frac{1}{2} i$ for any $z \in \mathbb{D}$. In this case we have

$$
\left|\left(g \oplus_{R} h\right)(z)\right|=\left|\frac{g(z)+h(z)}{1+g(z) h(z)}\right|=\frac{4}{3}
$$

Definition 3.3 (The relativistic product $\circ_{R}$ of generalized Fourier sequences) Let us consider two generalized Fourier sequences $Y_{n}(x, a)$ and $Z_{n}(x, a)$ defined by

$$
Y_{n}(x, a):=\sum_{j=0}^{n} C_{j}(n, a) e^{i \ell_{j}(n) x}, \quad Z_{n}(x, a):=\sum_{j=0}^{n} C_{j}(n, a) e^{i k_{j}(n) x}, \quad x \in \mathbb{R},
$$

with $\ell_{j}(n):=g(1-2 j / n)$ and $k_{j}(n):=h(1-2 j / n)$ for $h, g: \mathbb{D} \rightarrow \mathbb{D}$. Moreover, we assume that $h$ and $g$ satisfy the conditions $h((-1,1)) \subset(-1,1)$ and $g((-1,1)) \subset$ $(-1,1)$. The relativistic product of $Y_{n}(x, a)$ and $Z_{n}(x, a)$, denoted by $\circ_{R}$, is defined as

$$
\left(Y_{n} \circ_{R} Z_{n}\right)(x, a):=\sum_{j=0}^{n} C_{j}(n, a) e^{i\left(\ell_{j}(n) \oplus_{R} k_{j}(n)\right) x},
$$

where $\oplus_{R}$ is the relativistic sum (15).

Remark 3.4 When we consider the supershift notion in Definition 3.3, we do not have to assume that $f$ and $g$ are real valued on $(-1,1)$.

Definition 3.5 (The operator associated with the relativistic sum) Let $g$ and $h$ be holomorphic functions form $\mathbb{D}$ to $\mathbb{D}$. Let $A$ and $B$ be holomorphic functions from $\mathbb{D}$ to $\mathbb{C}$ defined by

$$
A(z):=g(z) h(z), \quad \text { and } \quad B(z):=g(z)+h(z),
$$

which have series expansions:

$$
A(z)=\sum_{p=0}^{\infty} a_{p} z^{p}, \quad B(z)=\sum_{q=0}^{\infty} b_{q} z^{q}
$$


Associated with $A$ and $B$ we define the operators

$$
A\left(\partial_{\xi}\right)=\sum_{p=0}^{\infty} a_{p} i^{-p} \partial_{\xi}^{p}, \quad B\left(\partial_{\xi}\right)=\sum_{q=0}^{\infty} b_{q} i^{-q} \partial_{\xi}^{q}
$$

and

$$
C\left(\partial_{\xi}\right)=B\left(\partial_{\xi}\right) \sum_{\ell=0}^{\infty}(-1)^{\ell} A^{\ell}\left(\partial_{\xi}\right) .
$$

The linear operator associated with the relativistic sum is defined as

$$
\mathcal{U}_{R}\left(x, \partial_{\xi}\right)=\sum_{m=0}^{\infty} \frac{(i x)^{m}}{m !} C\left(\partial_{\xi}\right)^{m}
$$

where $x \in \mathbb{R}$ is a parameter and $\xi \in \mathbb{C}$.

Remark 3.6 The operator $C\left(\partial_{\xi}\right)$, introduced in the Definition 3.5, is obtained by the formal substitution of $z$ with $\partial_{\xi}$ in the relativistic sum of $g$ and $h$. For we observe that

$$
\begin{aligned}
\left(g \oplus_{R} h\right)(z) & =\frac{g(z)+h(z)}{1+g(z) h(z)}=(g(z)+h(z)) \sum_{\ell=0}^{\infty}(-1)^{\ell}(g(z) h(z))^{\ell} \\
& =B(z) \sum_{\ell=0}^{\infty}(-1)^{\ell}(A(z))^{\ell} .
\end{aligned}
$$

The natural space of holomorphic functions on which the operator $\mathcal{U}_{R}\left(x, \partial_{\xi}\right)$ acts continuously is defined as follows.

Definition 3.7 Let $\alpha$ be a fixed positive number. We define the class $\mathcal{A}_{1, \alpha}$ to be the set of entire functions such that there exists $C>0$ for which

$$
|f(z)| \leq C \exp (\alpha|z|)
$$

Let $\left(f_{n}\right)_{n \in \mathbb{N}}, f_{0} \in \mathcal{A}_{1, \alpha}$. Then $f_{n} \rightarrow f_{0}$ in $\mathcal{A}_{1, \alpha}$ if

$$
\lim _{n \rightarrow \infty} \sup _{z \in \mathbb{C}}\left|\left(f_{n}(z)-f_{0}(z)\right) e^{-\alpha|z|}\right|=0
$$

We are now ready to prove our main result on the continuity of the infinite order differential operator $\mathcal{U}_{R}\left(x, \partial_{\xi}\right)$ associated with the relativistic sum.

Remark 3.8 In the Theorem 3.9 below we will study the continuity of the operator $\mathcal{U}_{R}\left(x, \partial_{\xi}\right)$ defined in the domains $\mathcal{A}_{1, \alpha}$ for $\alpha>0$. In the proof of Theorem 3.11 will be sufficient to consider the continuity of the operator $\mathcal{U}_{R}(x, \partial \xi)$ defined in $\mathcal{A}_{1,1}$. 
Theorem 3.9 Let $\alpha>0$ be fixed. Let $g, h: \mathbb{D} \rightarrow \mathbb{D}$ be holomorphic functions, let $\left(a_{p}\right)_{p \in \mathbb{N}_{0}},\left(b_{q}\right)_{q \in \mathbb{N}_{0}}$ be the sequences of complex numbers as in (18) and such that the conditions

$$
\sum_{p=0}^{\infty}\left|a_{p}\right|(2 e \alpha)^{p}:=c_{1}<1 \quad \text { and } \quad \sum_{q=0}^{\infty}\left|b_{q}\right|(2 e \alpha)^{q}:=c_{2}<+\infty
$$

hold. Then, the linear operator $\mathcal{U}_{R}\left(x, \partial_{\xi}\right)$, for $x \in \mathbb{R}$, defined in (21), acts continuously from $\mathcal{A}_{1, \alpha}$ to $\mathcal{A}_{1}$. Moreover, we have

$$
\left.\mathcal{U}_{R}\left(x, \partial_{\xi}\right) e^{i \lambda \xi}\right|_{\xi=0}=e^{i x\left(h \oplus_{R} g\right)(\lambda)}
$$

for $|\lambda|<\alpha$.

Proof The proof is split in steps.

STEP 1 . We study the action of the operator $C\left(\partial_{\xi}\right)=B\left(\partial_{\xi}\right) \sum_{\ell=0}^{\infty}(-1)^{\ell} A^{\ell}\left(\partial_{\xi}\right)$ on holomorphic functions belonging to $\mathcal{A}_{1, \alpha}$.

We first consider the operator $A\left(\partial_{\xi}\right)$ defined in (19). Using the formula (12) for the operators $A^{\ell}\left(\partial_{\xi}\right): \mathcal{A}_{1, \alpha} \rightarrow \mathcal{A}_{1}$, we have

$$
\begin{aligned}
& \sum_{\ell=0}^{\infty}(-1)^{\ell} A\left(\partial_{\xi}\right)^{\ell} f(\xi) \\
& \quad=\sum_{\ell=0}^{\infty}(-1)^{\ell} \sum_{p_{1}=0, \ldots, p_{\ell}=0}^{\infty} a_{p_{1}} \cdots a_{p_{\ell}} i^{-\left(p_{1}+\cdots+p_{\ell}\right)} \\
& \quad \sum_{j=0}^{\infty} f_{p_{1}+\cdots+p_{\ell}+j} \frac{\left(p_{1}+\cdots+p_{\ell}+j\right) !}{j !} \xi^{j}
\end{aligned}
$$

and the operator $C\left(\partial_{\xi}\right): \mathcal{A}_{1, \alpha} \rightarrow \mathcal{A}_{1}$, defined in (20), acts as

$$
C\left(\partial_{\xi}\right) f(\xi)=B\left(\partial_{\xi}\right) \sum_{\ell=0}^{\infty}(-1)^{\ell} A\left(\partial_{\xi}\right)^{\ell} f(\xi)=\sum_{q=0}^{\infty} b_{q} i^{-q} \partial_{\xi}^{q} \sum_{\ell=0}^{\infty}(-1)^{\ell} A\left(\partial_{\xi}\right)^{\ell} f(\xi)
$$

Using (24) it turns out that

$$
\begin{aligned}
C\left(\partial_{\xi}\right) f(\xi) & =\sum_{q=0}^{\infty} b_{q} i^{-q} \sum_{\ell=0}^{\infty}(-1)^{\ell} \sum_{p_{1}=0, \ldots, p_{\ell}=0}^{\infty} a_{p_{1}} \cdots a_{p_{\ell}} \\
& \times i^{-\left(p_{1}+\cdots+p_{\ell}\right)} \sum_{j=0}^{\infty} f_{p_{1}+\cdots+p_{\ell}+q+j} \frac{\left(p_{1}+\cdots+p_{\ell}+q+j\right) !}{j !} \xi^{j} .
\end{aligned}
$$


STEP 2. To compute $C\left(\partial_{\xi}\right)^{m}$ we need some more notation. By the formula (25), whenever we fix the index $m$ we have the following set of indexes: $q_{\mu}, \ell_{\mu}, p_{1}^{(\mu)}, \ldots, p_{\ell_{i}}^{(\mu)}$ for $\mu=1, \ldots, m$. We will prove, by induction, that:

$$
\begin{aligned}
C\left(\partial_{\xi}\right)^{m} f(\xi) & =\sum_{q_{1}=0}^{\infty} b_{q_{1}} i^{-q_{1}} \sum_{\ell_{1}=0}^{\infty}(-1)^{\ell_{1}} \sum_{p_{1}^{(1)}=0, \ldots, p_{\ell_{1}}^{(1)}=0}^{\infty} a_{p_{1}^{(1)}} \cdots a_{p_{\ell_{1}}^{(1)}} i^{-\left(p_{1}^{(1)}+\cdots+p_{\ell_{1}}^{(1)}\right)} \\
& \times \ldots \\
& \times \sum_{q_{m}=0}^{\infty} b_{q_{m}} i^{-q_{m}} \sum_{\ell_{m}=0}^{\infty}(-1)^{\ell_{m}} \sum_{p_{1}^{(m)}=0, \ldots, p_{\ell_{m}}^{(m)}=0}^{\infty} a_{p_{1}^{(m)}} \cdots a_{p_{\ell_{m}}^{(m)}} i^{-\left(p_{1}^{(m)}+\cdots+p_{\ell_{m}}^{(m)}\right)} \\
& \times \sum_{j=0}^{\infty} f_{\sum_{\mu=1}^{m}\left(p_{1}^{(\mu)}+\cdots+p_{\ell_{\mu}}^{(\mu)}+q_{\mu}\right)+j} \frac{\left(\sum_{\mu=1}^{m}\left(p_{1}^{(\mu)}+\cdots+p_{\ell_{\mu}}^{(\mu)}+q_{\mu}\right)+j\right) !}{j !} \xi^{j} .
\end{aligned}
$$

The first step (i.e., $m=1$ ) is described in the formula (25). We now proceed by induction. We suppose the formula holds to be true for $m>1$ and we show that it is true for $m+1$ :

$$
\begin{aligned}
& C\left(\partial_{\xi}\right)^{m+1} f(\xi)=C\left(\partial_{\xi}\right)\left(C\left(\partial_{\xi}\right)^{m} f(\xi)\right) \\
& =C\left(\partial_{\xi}\right)\left(\sum_{q_{1}=0}^{\infty} b_{q_{1}} i^{-q_{1}} \sum_{\ell_{1}=0}^{\infty}(-1)^{\ell_{1}} \sum_{p_{1}^{(1)}=0, \ldots, p_{\ell_{1}}^{(1)}=0}^{\infty} a_{p_{1}^{(1)}} \cdots a_{p_{\ell_{1}}^{(1)}} i^{-\left(p_{1}^{(1)}+\cdots+p_{\ell_{1}}^{(1)}\right)}\right. \\
& \times \ldots \\
& \times \sum_{q_{m}=0}^{\infty} b_{q_{m}} i^{-q_{m}} \sum_{\ell_{m}=0}^{\infty}(-1)^{\ell_{m}} \sum_{p_{1}^{(m)}=0, \ldots, p_{\ell_{m}}^{(m)}=0}^{\infty} a_{p_{1}^{(m)}} \cdots a_{p_{\ell_{m}}^{(m)} i^{-\left(p_{1}^{(m)}+\cdots+p_{\ell_{m}}^{(m)}\right)}} \\
& \left.\times \sum_{j=0}^{\infty} f_{\sum_{\mu=1}^{m}\left(p_{1}^{(\mu)}+\cdots+p_{\ell \mu}^{(\mu)}+q_{\mu}\right)+j} \frac{\left(\sum_{\mu=1}^{m}\left(p_{1}^{(\mu)}+\cdots+p_{\ell_{\mu}}^{(\mu)}+q_{\mu}\right)+j\right) !}{j !} \xi^{j}\right) \\
& =\sum_{q=0}^{\infty} b_{q} i^{-q} \sum_{\ell=0}^{\infty}(-1)^{\ell} \sum_{p_{1}=0, \ldots, p_{\ell}=0}^{\infty} a_{p_{1}} \cdots a_{p_{\ell}} i^{-\left(p_{1}+\cdots+p_{\ell}\right)} \\
& \times\left(\sum_{q_{1}=0}^{\infty} b_{q_{1}} i^{-q_{1}} \sum_{\ell_{1}=0}^{\infty}(-1)^{\ell_{1}} \sum_{p_{1}^{(1)}=0, \ldots, p_{\ell_{1}}^{(1)}=0}^{\infty} a_{p_{1}^{(1)}} \cdots a_{p_{\ell_{1}}^{(1)}} i^{-\left(p_{1}^{(1)}+\cdots+p_{\ell_{1}}^{(1)}\right)}\right. \\
& \times \ldots \\
& \times \sum_{q_{m}=0}^{\infty} b_{q_{m}} i^{-q_{m}} \sum_{\ell_{m}=0}^{\infty}(-1)^{\ell_{m}} \sum_{p_{1}^{(m)}=0, \ldots, p_{\ell_{m}}^{(m)}=0}^{\infty} a_{p_{1}^{(m)}} \cdots a_{p_{\ell_{m}}^{(m)}} i^{-\left(p_{1}^{(m)}+\cdots+p_{\ell_{m}}^{(m)}\right)}
\end{aligned}
$$




$$
\begin{aligned}
& \times \sum_{j=0}^{\infty} f_{p_{1}+\cdots+p_{l}+q+\sum_{\mu=1}^{m}\left(p_{1}^{(\mu)}+\cdots+p_{\ell_{\mu}}^{(\mu)}+q_{\mu}\right)+j} \\
& \left.\times \frac{\left(p_{1}+\cdots+p_{l}+q+\sum_{\mu=1}^{m}\left(p_{1}^{(\mu)}+\cdots+p_{\ell_{\mu}}^{(\mu)}+q_{\mu}\right)+j\right) !}{j !} \xi^{j}\right),
\end{aligned}
$$

where in the last equality we have used (25). After renaming the indexes and letting $\mu$ varying from 1 to $m+1$, we obtain

$$
\begin{aligned}
& C\left(\partial_{\xi}\right)^{m+1} f(\xi)=\sum_{q_{1}=0}^{\infty} b_{q_{1}} i^{-q_{1}} \sum_{\ell_{1}=0}^{\infty}(-1)^{\ell_{1}} \sum_{p_{1}^{(1)}=0, \ldots, p_{\ell_{1}}^{(1)}=0}^{\infty} a_{p_{1}^{(1)}} \cdots a_{p_{\ell_{1}}^{(1)}}-\left(p_{1}^{(1)}+\cdots+p_{\ell_{1}}^{(1)}\right) \\
& \quad \times \ldots \\
& \quad \times \sum_{q_{m+1}=0}^{\infty} b_{q_{m+1}} i^{-q_{m+1}} \sum_{\ell_{m+1}=0}^{\infty}(-1)^{\ell_{m+1}} \\
& \quad \sum_{1}^{\infty} a_{p_{1}^{(m+1)}}^{\infty} \cdots a_{p_{\ell_{m+1}(m+1)} i^{-\left(p_{1}^{(m+1)}+\cdots+p_{\left.\ell_{m+1}^{(m+1)}\right)}\right.}} \sum^{\infty} \sum_{j=0}^{\infty} f_{\sum_{\mu=1}^{m+1}\left(p_{1}^{(\mu)}+\cdots+p_{\ell_{\mu}}^{(\mu)}+q_{\mu}\right)+j} \frac{\left(\sum_{\mu=1}^{m+1}\left(p_{1}^{(\mu)}+\cdots+p_{\ell_{\mu}}^{(\mu)}+q_{\mu}\right)+j\right) !}{j !} \xi^{j} .
\end{aligned}
$$

Thus formula (26) are proved.

STEP 3. We show that $\mathcal{U}_{R}\left(x, \partial_{\xi}\right)$, defined in (21), acts continuously from $\mathcal{A}_{1, \alpha}$ to $\mathcal{A}_{1}$.

The formula (26), which gives the explicit expression of $C\left(\partial_{\xi}\right)^{m}$, will be crucial to study the continuity of $\mathcal{U}_{R}\left(x, \partial_{\xi}\right)$. In fact, applying the operator $\mathcal{U}_{R}\left(x, \partial_{\xi}\right)$ to holomorphic functions such that $|f(z)| \leq C \exp (\alpha|z|)$ we have:

$$
\begin{aligned}
\mathcal{U}_{R}\left(x, \partial_{\xi}\right) f(\xi)= & \sum_{m=0}^{\infty} \frac{(i x)^{m}}{m !} C\left(\partial_{\xi}\right)^{m} f(\xi) \\
& =\sum_{m=0}^{\infty} \frac{(i x)^{m}}{m !} \sum_{q_{1}=0}^{\infty} b_{q_{1}} i^{-q_{1}} \sum_{\ell_{1}=0}^{\infty}(-1)^{\ell_{1}} \\
& \sum_{p_{1}^{(1)}=0, \ldots, p_{\ell_{1}}^{(1)}=0}^{\infty} a_{p_{1}^{(1)}} \cdots a_{p_{\ell_{1}}(1)} i^{-\left(p_{1}^{(1)}+\cdots+p_{\ell_{1}}^{(1)}\right)} \\
& \times \ldots \\
& \times \sum_{q_{m}=0}^{\infty} b_{q_{m}} i^{-q_{m}} \sum_{\ell_{m}=0}^{\infty}(-1)^{\ell_{m}} \sum_{p_{1}^{(m)}=0, \ldots, p_{\ell_{m}}^{(m)}=0}^{\infty} a_{p_{1}^{(m)}} \cdots a_{p_{\ell_{m}}^{(m)}} i^{-\left(p_{1}^{(m)}+\cdots+p_{\ell_{m}}^{(m)}\right)} \\
& \times \sum_{j=0}^{\infty} f_{\sum_{\mu=1}^{m}\left(p_{1}^{(\mu)}+\cdots+p_{\ell_{\mu}}^{(\mu)}+q_{\mu}\right)+j} \frac{\left(\sum_{\mu=1}^{m}\left(p_{1}^{(\mu)}+\cdots+p_{\ell_{\mu}}^{(\mu)}+q_{\mu}\right)+j\right) !}{j !} \xi^{j} .
\end{aligned}
$$


Taking the modulus of both sides in the previous equality, we obtain

$$
\begin{aligned}
\left|\mathcal{U}_{R}\left(x, \partial_{\xi}\right) f(\xi)\right| \leq & C_{f} \sum_{m=0}^{\infty} \frac{|x|^{m}}{m !} \sum_{q_{1}=0}^{\infty}\left|b_{q_{1}}\right| \sum_{\ell_{1}=0}^{\infty} \sum_{p_{1}^{(1)}=0, \ldots, p_{\ell_{1}}^{(1)}=0}^{\infty}\left|a_{p_{1}^{(1)}}\right| \cdots\left|a_{p_{\ell_{1}}^{(1)}}\right| \\
& \times \ldots \\
& \times \sum_{q_{m}=0}^{\infty}\left|b_{q_{m}}\right| \sum_{\ell_{m}=0}^{\infty} \sum_{p_{1}^{(m)}=0, \ldots, p_{\ell_{m}}^{(m)}=0}^{\infty}\left|a_{p_{1}^{(m)}}\right| \cdots\left|a_{p_{\ell_{m}}^{(m)}}\right| \\
& \times \sum_{j=0}^{\infty} \frac{b_{f}^{\sum_{\mu=1}^{m}\left(p_{1}^{(\mu)}+\cdots+p_{\ell_{\mu}}^{(\mu)}+q_{\mu}\right)+j}}{\left(\sum_{\mu=1}^{m}\left(p_{1}^{(\mu)}+\cdots+p_{\ell_{\mu}}^{(\mu)}+q_{\mu}\right)+j\right) !} \\
& \frac{\left(\sum_{\mu=1}^{m}\left(p_{1}^{(\mu)}+\cdots+p_{\ell_{\mu}}^{(\mu)}+q_{\mu}\right)+j\right) !}{j !}|\xi|^{j}
\end{aligned}
$$

where we have used the estimate

$$
\left|f_{\sum_{\mu=1}^{m}\left(p_{1}^{(\mu)}+\cdots+p_{\ell_{\mu}}^{(\mu)}+q_{\mu}\right)+j}\right| \leq C_{f} \frac{b_{f}^{\sum_{\mu=1}^{m}\left(p_{1}^{(\mu)}+\cdots+p_{\ell_{\mu}}^{(\mu)}+q_{\mu}\right)+j}}{\left(\sum_{\mu=1}^{m}\left(p_{1}^{(\mu)}+\cdots+p_{\ell_{\mu}}^{(\mu)}+q_{\mu}\right)+j\right) !} .
$$

Since $f \in \mathcal{A}_{1, \alpha}$ we have that

$$
b_{f}=2 e \alpha
$$

where we have used Lemma 2.2 with $p=1$ (see also Remark 2.3 with $p=1$ ).

After some computations we get

$$
\begin{aligned}
\left|\mathcal{U}_{R}\left(x, \partial_{\xi}\right) f(\xi)\right| & \leq \sum_{m=0}^{\infty} \frac{|x|^{m}}{m !} \sum_{q_{1}=0}^{\infty}\left|b_{q_{1}}\right| b_{f}^{q_{1}} \sum_{\ell_{1}=0}^{\infty} \sum_{p_{1}^{(1)}=0, \ldots, p_{\ell_{1}}^{(1)}=0}^{\infty}\left|a_{p_{1}^{(1)}}\right| \cdots\left|a_{p_{\ell_{1}}^{(1)}}\right| b_{f}^{p_{1}^{(1)}+\cdots+p_{\ell_{1}}^{(1)}} \\
& \times \ldots \\
& \times \sum_{q_{m}=0}^{\infty}\left|b_{q_{m}}\right| b_{f}^{q_{m}} \sum_{\ell_{m}=0}^{\infty} \sum_{p_{1}^{(m)}=0, \ldots, p_{\ell_{m}}^{(m)}=0}^{\infty}\left|a_{p_{1}^{(m)}}\right| \cdots\left|a_{p_{\ell_{m}}^{(m)}}\right| b_{f}^{p_{1}^{(m)}+\cdots+p_{\ell_{m}}^{(m)}} \\
& \times \sum_{j=0}^{\infty} \frac{b_{f}^{k}}{j !}|\xi|^{j} .
\end{aligned}
$$

Keeping in mind that $b_{f}=2 e \alpha$ and recalling the assumptions (23) on the coefficients $a_{p}$ 's and $b_{q}$ 's, there exist two positive numbers $c_{1}<1$ and $c_{2}$ such that

$$
\sum_{p=0}^{\infty}\left|a_{p}\right| b_{f}^{p}=c_{1} \quad \text { and } \quad \sum_{q=0}^{\infty}\left|b_{q}\right| b_{f}^{q}=c_{2} .
$$


Thus we obtain

$$
\begin{aligned}
\left|\mathcal{U}_{R}\left(x, \partial_{\xi}\right) f(\xi)\right| & \leq C_{f} e^{b_{f}|\xi|} \sum_{m=0}^{\infty} \frac{|x|^{m}}{m !}\left(c_{2}\right)^{m} \sum_{\ell_{1}=0}^{\infty}\left(c_{1}\right)^{\ell_{1}} \times \ldots \times \sum_{\ell_{m}=0}^{\infty}\left(c_{1}\right)^{\ell_{m}} \\
& \leq C_{f} e^{b_{f}|\xi|} \sum_{m=0}^{\infty} \frac{|x|^{m}}{m !}\left(\frac{c_{2}}{1-c_{1}}\right)^{m}
\end{aligned}
$$

and finally, setting

$$
k=\frac{c_{2}}{1-c_{1}}
$$

we have

$$
\left|\mathcal{U}_{R}\left(x, \partial_{\xi}\right) f(\xi)\right| \leq C_{f} e^{b_{f}|\xi|+k|x|},
$$

where $b_{f}=2 e \alpha$. The previous inequality means that $\mathcal{U}_{R}\left(x, \partial_{\xi}\right) f(\xi) \in \mathcal{A}_{1}$ and also that $\mathcal{U}_{R}\left(x, \partial_{\xi}\right)$ is continuous from $\mathcal{A}_{1, \alpha}$ to $\mathcal{A}_{1}$. Precisely, taking $f_{n}$ such that $f_{n} \rightarrow 0$ the estimate

$$
\left|\mathcal{U}_{R}\left(x, \partial_{\xi}\right) f_{n}(\xi)\right| \leq C_{f_{n}} e^{b_{n}|\xi|+k|x|}
$$

with the bounds $b_{n} \leq 2 e \alpha$, shows that $U\left(x, \partial_{\xi}\right) f_{n}(\xi) \rightarrow 0$. Finally we have for $|\lambda|<\alpha$

$$
\begin{aligned}
& \left.\left(\mathcal{U}_{R}(x, \partial \xi) e^{i \lambda \xi}\right)\right|_{\xi=0}=\left.\left(\sum_{m=0}^{\infty} \frac{(i x)^{m}}{m !} C\left(\partial_{\xi}\right)^{m} e^{i \lambda \xi}\right)\right|_{\xi=0} \\
& =\left(\sum_{m=0}^{\infty} \frac{(i x)^{m}}{m !} \sum_{q_{1}=0}^{\infty} b_{q_{1}} i^{-q_{1}} \sum_{\ell_{1}=0}^{\infty}(-1)^{\ell_{1}} \sum_{p_{1}^{(1)}=0, \ldots, p_{\ell_{1}}^{(1)}=0}^{\infty} a_{p_{1}^{(1)}} \cdots a_{p_{\ell_{1}}^{(1)}} i^{-\left(p_{1}^{(1)}+\cdots+p_{\ell_{1}}^{(1)}\right)}\right. \\
& \times \ldots \\
& \times \sum_{q_{m}=0}^{\infty} b_{q_{m}} i^{-q_{m}} \sum_{\ell_{m}=0}^{\infty}(-1)^{\ell_{m}} \sum_{p_{1}^{(m)}=0, \ldots, p_{\ell_{m}}^{(m)}=0}^{\infty} a_{p_{1}^{(m)}} \cdots a_{p_{\ell_{m}}^{(m)}} i^{-\left(p_{1}^{(m)}+\cdots+p_{\ell_{m}}^{(m)}\right)} \\
& \left.\times \sum_{j=0}^{\infty} \frac{(i \lambda)^{\sum_{\mu=1}^{m}\left(p_{1}^{(\mu)}+\cdots+p_{\ell_{\mu}}^{(\mu)}+q_{\mu}\right)+j}}{\left(\sum_{\mu=1}^{m}\left(p_{1}^{(\mu)}+\cdots+p_{\ell_{\mu}}^{(\mu)}+q_{\mu}\right)+j\right) !} \frac{\left(\sum_{\mu=1}^{m}\left(p_{1}^{(\mu)}+\cdots+p_{\ell_{\mu}}^{(\mu)}+q_{\mu}\right)+j\right) !}{j !} \xi^{j}\right)\left.\right|_{\xi=0} \\
& =\sum_{m=0}^{\infty} \frac{(i x)^{m}}{m !} \sum_{q_{1}=0}^{\infty} b_{q_{1}} i^{-q_{1}} \sum_{\ell_{1}=0}^{\infty}(-1)^{\ell_{1}} \sum_{p_{1}^{(1)}=0, \ldots, p_{\ell_{1}}^{(1)}=0}^{\infty} a_{p_{1}^{(1)}} \cdots a_{p_{\ell_{1}}^{(1)}} i^{-\left(p_{1}^{(1)}+\cdots+p_{\ell_{1}}^{(1)}\right)} \\
& \times \ldots \\
& \times \sum_{q_{m}=0}^{\infty} b_{q_{m}} i^{-q_{m}} \sum_{\ell_{m}=0}^{\infty}(-1)^{\ell_{m}} \sum_{p_{1}^{(m)}=0, \ldots, p_{\ell_{m}}^{(m)}=0}^{\infty} a_{p_{1}^{(m)}} \cdots a_{p_{\ell_{m}}^{(m)}} i^{-\left(p_{1}^{(m)}+\cdots+p_{\ell_{m}}^{(m)}\right)}
\end{aligned}
$$




$$
\begin{aligned}
& \times(i \lambda)^{\sum_{\mu=1}^{m} p_{1}^{(\mu)}+\cdots+p_{\ell_{\mu}}^{(\mu)}+q_{\mu}} \\
& =\sum_{m=0}^{\infty} \frac{(i x)^{m}}{m !}\left(\sum_{q=0}^{\infty} b_{q} \sum_{\ell=0}^{\infty}(-1)^{\ell} \sum_{p_{1}=0, \ldots, p_{\ell}=0}^{\infty} a_{p_{1}} \cdots a_{p_{\ell}} \lambda^{p_{1}+\cdots+p_{\ell}+q}\right)^{m}=e^{i x\left(h \oplus_{R} g\right)(\lambda)} .
\end{aligned}
$$

Remark 3.10 In the case of the relativistic sum, we have to re-scale the interval $(-1,1)$ in the definition of superoscillations and/or supershift property because here we work in the unit ball in $\mathbb{C}$. So we take $\varepsilon \in(0,1)$ and the interval $(-1,1)$ is replaced by the interval $(-\varepsilon, \varepsilon)$ when we set $\ell_{j}(n):=g(\varepsilon(1-2 j / n))$ and $k_{j}(n):=h(\varepsilon(1-2 j / n))$.

We are ready to state the main result on the relativistic product.

Theorem 3.11 Let $Y_{n}(x, a)$ and $Z_{n}(x, a)$ be two generalized Fourier sequences for $x, a \in \mathbb{R}$, with $\ell_{j}(n):=g(\varepsilon(1-2 j / n))$ and $k_{j}(n):=h(\varepsilon(1-2 j / n))$ as in Definition 3.3 for $\varepsilon \in(0,1)$ and such that conditions $(23)$ of Theorem 3.9 are satisfied for $\alpha=1$. Let $\left(Y_{n} \circ_{R} Z_{n}\right)(x, a)$ be the relativistic product. Then we have

$$
\lim _{n \rightarrow \infty}\left(Y_{n} \circ_{R} Z_{n}\right)(x, a)=e^{i x\left(g \oplus_{R} h\right)(\varepsilon a)}
$$

for any $|\varepsilon a|<1$.

Remark 3.12 The Theorem 3.11 also works in the case of sequences that satisfy the supershift property (in particular in this case $|a|>1$ ) but they are not necessarily generalized Fourier sequences.

Proof For every $a \in \mathbb{R}$ and $\varepsilon \in(0,1)$ such that $|\varepsilon a|<1$, we consider

$$
\left(Y_{n} \circ_{R} Z_{n}\right)(x, a)=\sum_{j=0}^{n} C_{j}(n, a) e^{i x\left(g \oplus_{R} h\right)(\varepsilon(1-2 j / n))} .
$$

We define the operator $\mathcal{U}_{R}\left(x, \partial_{\xi}\right)$ as in (21) and we consider its action over the space $\mathcal{A}_{1,1}$ (see Remark 3.8). By Theorem 3.9 we obtain the relations

$$
e^{i x\left(g \oplus_{R} h\right)(\varepsilon(1-2 j / n))}=\left.\mathcal{U}_{R}\left(x, \partial_{\xi}\right) e^{i \xi(\varepsilon(1-2 j / n))}\right|_{\xi=0}
$$

and

$$
\left.\mathcal{U}_{R}\left(x, \partial_{\xi}\right) e^{i \varepsilon a \xi}\right|_{\xi=0}=e^{i x\left(h \oplus_{R} g\right)(\varepsilon a)} .
$$


Thus we have

$$
\begin{aligned}
& \lim _{n \rightarrow \infty}\left(Y_{n} \circ_{R} Z_{n}\right)(x, a)=\lim _{n \rightarrow \infty} \sum_{j=0}^{n} C_{j}(n, a) e^{i x\left(h \oplus_{R} g\right)(\varepsilon(1-2 j / n))} \\
& \left.\stackrel{(30)}{=} \lim _{n \rightarrow \infty} \sum_{j=0}^{n} C_{j}(n, a) \mathcal{U}_{R}\left(x, \partial_{\xi}\right) e^{i \xi(\varepsilon(1-2 j / n))}\right|_{\xi=0} \\
& =\left.\lim _{n \rightarrow \infty} \mathcal{U}_{R}\left(x, \partial_{\xi}\right) \sum_{j=0}^{n} C_{j}(n, a) e^{i \xi(\varepsilon(1-2 j / n))}\right|_{\xi=0}
\end{aligned}
$$

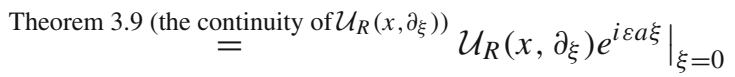

$$
\begin{aligned}
& \stackrel{(31)}{=} e^{i x\left(h \oplus_{R} g\right)(\varepsilon a)}
\end{aligned}
$$

where we have assumed $|\varepsilon a|<1$.

\section{Blaschke products and superoscillations}

In this section we will consider a new type of infinite order differential operators that will allow us to study generalized Fourier series defined by Blaschke products. Let $\left(b_{n}\right)_{n=1}^{M}$ be a finite sequence of complex numbers such that $0<\left|b_{i}\right|<1$ for $i=1, \ldots, M$. A finite Blaschke product is defined for $|z|<1$ by

$$
B(z):=\prod_{q=1}^{M}\left(\frac{b_{q}-z}{1-\bar{b}_{q} z}\right)=\prod_{q=1}^{M}\left(b_{q}-z\right) \sum_{p=0}^{\infty}\left(\bar{b}_{q} z\right)^{p},
$$

where the last equality holds in the unit disc since $\left|\bar{b}_{n} z\right|<1$. Evidently, the product of two finite Blaschke products is still a finite Blaschke product.

In the sequel we will study the limit of generalized Fourier sequences of the form:

$$
Y_{n}(x, a):=\sum_{j=0}^{n} C_{j}(n, a) e^{i B(\varepsilon(1-2 j / n)) x}, \text { for } \varepsilon \in(0,1),
$$

for $a \in \mathbb{R}$ such that $\varepsilon|a|<1$ and $B$ is a finite Blaschke product. In order to compute the limit for $n \rightarrow \infty$ we need the following infinite order differential operators formally obtained by the substitution of $z$ with $\partial_{\xi}$ in the series expansion (32).

Definition 4.1 Let $\left(b_{i}\right)_{i=1}^{M}$ be a finite sequence of complex numbers such that $0<$ $\left|b_{i}\right|<1$ for $i=1, \ldots, M$. Let $B(z)$ be a finite Blaschke products as in (32). We define the formal operators

$$
\mathcal{U}_{B}(x, \xi):=\sum_{m=0}^{\infty} \frac{(i x)^{m}}{m !} B^{m}\left(\partial_{\xi}\right) \quad \text { where } \quad B\left(\partial_{\xi}\right)=\prod_{q=1}^{M} \mathcal{V}_{q}\left(\partial_{\xi}\right)
$$


and

$$
\mathcal{V}_{q}\left(\partial_{\xi}\right):=\left(b_{q}-i^{-1} \partial_{\xi}\right) \sum_{p=0}^{\infty} \bar{b}_{q}^{p} i^{-p} \partial_{\xi}^{p}=b_{q} \sum_{p=0}^{\infty} \bar{b}_{q}^{p} i^{-p} \partial_{\xi}^{p}-\sum_{p=0}^{\infty} \bar{b}_{q}^{p} i^{-p-1} \partial_{\xi}^{p+1}
$$

We set $\mathcal{M}_{M}:=\{0,1\}^{M}$. Given an element $s \in \mathcal{M}_{M}$ we denote by $s_{i}$ the element in the $i$-th position of the vector $s$ and we denote by $s^{c}$ the vector in $\mathcal{M}_{M}$ defined by $\left(s^{c}\right)_{i}=1-s_{i}$.

Lemma 4.2 Let $(R,+, \cdot)$ be a ring. Then for any positive integer $M$ we have

$$
\prod_{i=1}^{M}\left(A_{i}^{(0)}+A_{i}^{(1)}\right)=\sum_{s \in \mathcal{M}_{M}} \prod_{i=1}^{M} A_{i}^{\left(s_{i}\right)}=\sum_{p=0}^{M} \sum_{\substack{s \in \mathcal{M}_{M} \\|s|=p}} \prod_{i=1}^{M} A_{i}^{\left(s_{i}\right)}
$$

where $A_{i}^{\left(s_{i}\right)} \in R$ and $|s|=\sum_{i=1}^{M} s_{i}$.

Proof We proceed by induction over $M$. The first step is trivial. We suppose that the formula (35) holds to be true for $M$ then for $M+1$ we have

$$
\begin{aligned}
\prod_{i=1}^{M+1}\left(A_{i}^{(0)}+A_{i}^{(1)}\right) & =\prod_{i=1}^{M}\left(A_{i}^{(0)}+A_{i}^{(1)}\right)\left(A_{M+1}^{(0)}+A_{M+1}^{(1)}\right) \\
& =\sum_{s \in \mathcal{M}_{M}} \prod_{i=1}^{M} A_{i}^{\left(s_{i}\right)}\left(A_{M+1}^{(0)}+A_{M+1}^{(1)}\right) \\
& =\sum_{s \in \mathcal{M}_{M}} \prod_{i=1}^{M} A_{i}^{\left(s_{i}\right)} A_{M+1}^{(0)}+\prod_{i=1}^{M} A_{i}^{\left(s_{i}\right)} A_{M+1}^{(1)} \\
& =\sum_{s \in \mathcal{M}_{M+1}} \prod_{i=1}^{M+1} A_{i}^{\left(s_{i}\right)} .
\end{aligned}
$$

Thus by induction the formula (35) holds for any $M$.

For the sake of simplicity, from now on we refer to the set $\mathcal{M}_{M}$ as $\mathcal{M}$.

Theorem 4.3 Let $\left(b_{i}\right)_{i=1}^{M}$ be a finite sequence of complex numbers such that $0<\left|b_{i}\right|<$ 1 for $i=1, \ldots, M$. Let $B(z)$ be the finite Blaschke product (32). Let $b$ be a positive constant such that $b<\min _{j=1, \ldots, M} \frac{1}{\left|b_{j}\right|}$. Then the operator

$$
\mathcal{U}_{B}\left(x, \partial_{\xi}\right)=\sum_{m=0}^{\infty} \frac{(i x)^{m}}{m !} B^{m}\left(\partial_{\xi}\right)
$$


in Definition 4.1 acts continuously from $\mathcal{A}_{1, \alpha}$ to $\mathcal{A}_{1}$ where $\alpha=\frac{b}{2 e}$. Moreover, we have that

$$
\left.\mathcal{U}_{B}\left(x, \partial_{\xi}\right) e^{i \lambda \xi}\right|_{\xi=0}=e^{i x B(\lambda)}
$$

for $|\lambda|<\min (\alpha, 1)$.

Proof Recalling (33), (34) we write

$$
B\left(\partial_{\xi}\right)=\prod_{q=1}^{M} \mathcal{V}_{q}\left(\partial_{\xi}\right)
$$

We observe that $\mathcal{V}_{q}\left(\partial_{\xi}\right)=A_{q}^{(0)}\left(\partial_{\xi}\right)+A_{q}^{(1)}\left(\partial_{\xi}\right)$ where

$$
A_{q}^{(0)}\left(\partial_{\xi}\right):=b_{q} \sum_{p=0}^{\infty} \bar{b}_{q}^{p} i^{-p} \partial_{\xi}^{p} \quad \text { and } \quad A_{q}^{(1)}\left(\partial_{\xi}\right):=-\sum_{p=0}^{\infty} \bar{b}_{q}^{p} i^{-p-1} \partial_{\xi}^{p+1}
$$

Using Lemma 4.2 we can write

$$
B\left(\partial_{\xi}\right)=\sum_{p=0}^{M} \sum_{\substack{s \in \mathcal{M} \\|s|=p}} \prod_{i=1}^{M} A_{i}^{\left(s_{i}\right)}\left(\partial_{\xi}\right)
$$

We fix an element $f \in A_{1, \alpha}$ and $s \in \mathcal{M}$ and we observe that

$$
\begin{aligned}
& \left(\prod_{i=1}^{M} A_{i}^{\left(s_{i}\right)}\left(\partial_{\xi}\right)\right) f(\xi)=\left(\prod_{i=1}^{M} b_{q_{i}}^{s_{i}^{c}}(-1)^{|s|} \sum_{p_{i}=0}^{\infty} \bar{b}_{q_{i}}^{p_{i}} i^{-p-s_{i}} \partial_{\xi}^{p_{i}+s_{i}}\right) f(\xi) \\
& =(-1)^{|s|}\left(b_{q_{1}}^{s_{1}^{c}} \ldots b_{q_{M}}^{s_{M}^{c}}\right) \sum_{p_{1}=0, \ldots, p_{M}=0}^{\infty} \bar{b}_{q_{1}}^{p_{1}} \cdots \bar{b}_{q_{M}}^{p_{M}} i^{-\left(p_{1}+\cdots+p_{M}+|s|\right)} \\
& \quad \times \sum_{j=0}^{\infty} f_{j+p_{1}+\cdots+p_{M}+|s|} \frac{\left(j+p_{1}+\cdots+p_{M}+|s|\right) !}{j !} \xi^{j} .
\end{aligned}
$$

Note that we have used the elements of the vector $s^{c}$ as exponents of the $b_{q}$ 's in the previous formula since they appear as factors in $A_{q}^{(0)}$ but not in $A_{q}^{(1)}$. Taking the absolute value of both sides in the previous formula and using the fact that $\left|f_{j}\right| \leq C_{f} \frac{b^{j}}{j !}$ we obtain 


$$
\begin{aligned}
\left|\left(\prod_{i=1}^{M} A_{i}^{\left(s_{i}\right)}\left(\partial_{\xi}\right)\right) f(\xi)\right| & \leq C_{f} \sum_{p_{1}=0, \ldots, p_{M}=0}^{\infty}\left|\bar{b}_{q_{1}}\right|^{p_{1}} \cdots\left|\bar{b}_{q_{M} \mid}\right|^{p_{M}} \sum_{j=0}^{\infty} \frac{b^{j+p_{1}+\cdots+p_{M}+|s|}}{j !}|\xi|^{j} \\
& \leq C_{f}\left(\sum_{p_{1}=0}^{\infty}\left|\bar{b}_{q_{1}}\right|^{p_{1}} b^{p_{1}}\right) \cdots\left(\sum_{p_{M}=0}^{\infty}\left|\bar{b}_{q_{M}}\right|^{p_{M}} b^{p_{M}}\right) b^{|s|} \sum_{j=0}^{\infty} \frac{b^{j}}{j !}|\xi|^{j} \\
& \leq C_{f} C^{M} b^{|s|} e^{b \xi},
\end{aligned}
$$

where in the third inequality we have used the fact that $b$ is in the set of the convergence of the series $\sum_{p=0}^{\infty}\left|b_{q_{i}}\right|^{p} x^{p}$ for any $i=1, \ldots, M$. Thus we obtain

$$
\begin{aligned}
\left|B\left(\partial_{\xi}\right) f(\xi)\right| & \leq C_{f} C^{M} e^{b \xi} \sum_{p=0}^{M} b^{p} \sum_{s \in \mathcal{M},|s|=p} 1 \\
& =C_{f} C^{M} e^{b \xi} \sum_{p=0}^{M}\left(\begin{array}{c}
M \\
p
\end{array}\right) b^{p} \\
& =C_{f} C^{M} e^{b \xi}(1+b)^{M} .
\end{aligned}
$$

Moreover, we have

$$
\begin{aligned}
& \left|B^{m}\left(\partial_{\xi}\right) f(x)\right|=\left|\left(\prod_{j=1}^{m} \sum_{s^{j} \in \mathcal{M}} \prod_{i=1}^{M} A_{i}^{\left(s_{i}^{j}\right)}\left(\partial_{\xi}\right)\right) f(\xi)\right| \\
& =\left|\sum_{s^{1} \in \mathcal{M}} \cdots \sum_{s^{m} \in \mathcal{M}} \prod_{j=1}^{m} \prod_{i=1}^{M} A_{i}^{\left(s_{i}^{j}\right)}\left(\partial_{\xi}\right) f(\xi)\right| \\
& =\mid \sum_{s^{1} \in \mathcal{M}} \cdots \sum_{s^{m} \in \mathcal{M}}\left(b_{q_{1}}^{\left(s_{1}^{1}\right)^{c}} \ldots b_{q_{M}}^{\left(s_{M}^{1}\right)^{c}}\right) \cdots\left(b_{q_{1}}^{\left(s_{1}^{m}\right)^{c}} \ldots b_{q_{M}}^{\left(s_{M}^{m}\right)^{c}}\right) \\
& \left(\sum_{p_{1}^{(1)}=0, \ldots, p_{M}^{(1)}=0}^{\infty} \bar{b}_{q_{1}}^{p_{1}^{(1)}} \cdots \bar{b}_{q_{M}}^{p_{M}^{(1)}}\right) \times \ldots \times\left(\sum_{p_{1}^{(m)}=0, \ldots, p_{M}^{(m)}=0}^{\infty} \bar{b}_{q_{1}}^{p_{1}^{(m)}} \cdots \bar{b}_{q_{M}}^{p_{m}^{(m)}}\right) \\
& \sum_{j=0}^{\infty} f_{j+\sum_{\mu=1}^{m} p_{1}^{(\mu)}+\cdots+p_{M}^{(\mu)}+\left|s^{\mu}\right|} \frac{\left(j+\sum_{\mu=1}^{m} p_{1}^{(\mu)}+\cdots+p_{M}^{(\mu)}+\left|s^{\mu}\right|\right) !}{j !} \xi^{j} \mid \\
& \leq C_{f} \sum_{s^{1} \in \mathcal{M}} \ldots \sum_{s^{m} \in \mathcal{M}}\left(\sum_{p_{1}^{(1)}=0, \ldots, p_{M}^{(1)}=0}^{\infty}\left|\bar{b}_{q_{1}}\right|_{1}^{(1)}|b|^{p_{1}^{(1)}} \cdots\left|\bar{b}_{q_{M}}\right|^{p_{M}^{(1)}}|b|^{p_{M}^{(1)}}\right) \times \ldots \\
& \times\left(\sum_{p_{1}^{(m)}=0, \ldots, p_{M}^{(m)}=0}^{\infty}\left|\bar{b}_{q_{1}}\right|^{p_{1}^{(m)}}|b|^{p_{1}^{(m)}} \cdots\left|\bar{b}_{q_{M}}\right|^{p_{M}^{(m)}}|b|^{p_{M}^{(m)}}\right) b^{\sum_{\mu=1}^{m}\left|s^{\mu}\right|} \sum_{j=0}^{\infty} \frac{b^{j}}{j !}|\xi|^{j} \\
& \leq C_{f} C^{m M}(1+b)^{m M} e^{b \xi} \text {. }
\end{aligned}
$$


We deduce

$$
\begin{aligned}
\left|\mathcal{U}_{B}\left(x, \partial_{\xi}\right) f(\xi)\right| \leq & \sum_{m=0}^{\infty} \frac{|x|^{m}}{m !}\left|B^{m}\left(\partial_{\xi}\right) f(\xi)\right| \\
& \leq C_{f} \sum_{m=0}^{\infty} \frac{|x|^{m}}{m !} C^{m M}(1+b)^{m M} \sum_{j=0} \frac{b^{j}|\xi|^{j}}{j !} \\
& =C_{f} e^{|x|\left(1+b^{M}\right) C^{M}} e^{b|\xi|}
\end{aligned}
$$

The previous inequality means that $\mathcal{U}_{B}\left(x, \partial_{\xi}\right) f(\xi) \in \mathcal{A}_{1}$ and also that $\mathcal{U}_{B}(x, \partial \xi)$ is continuous from $\mathcal{A}_{1, \alpha}$ to $A_{1}$. Finally we have that

$$
\begin{aligned}
& \left.\mathcal{U}_{B}\left(x, \partial_{\xi}\right) e^{i \lambda \xi}\right|_{\xi=0}=\left.\left(\sum_{m=0}^{\infty} \frac{(i x)^{m}}{m !} B^{m}\left(\partial_{\xi}\right) e^{i \lambda \xi}\right)\right|_{\xi=0} \\
& =\left(\sum_{m=0}^{\infty} \frac{(i x)^{m}}{m !} \sum_{s^{1} \in \mathcal{M}} \cdots \sum_{s^{m} \in \mathcal{M}}\left(b_{q_{1}}^{\left(s_{1}^{1}\right)^{c}} \ldots b_{q_{M}}^{\left(s_{M}^{1}\right)^{c}}\right) \cdots\left(b_{q_{1}}^{\left(s_{1}^{m}\right)^{c}} \ldots b_{q_{M}}^{\left(s_{M}^{m}\right)^{c}}\right)(-1)^{\sum_{\mu=1}^{m}\left|s^{\mu}\right|}\right. \\
& \times\left(\sum_{p_{1}^{(1)}=0, \ldots, p_{M}^{(1)}=0}^{\infty} \bar{b}_{q_{1}}^{p_{1}^{(1)}} \cdots \bar{b}_{q_{M}}^{p_{M}^{(1)}}\right) \times \ldots \\
& \times\left(\sum_{p_{1}^{(m)}=0, \ldots, p_{M}^{(m)}=0}^{\infty} \bar{b}_{q_{1}}^{p_{1}^{(m)}} \cdots \bar{b}_{q_{M}}^{p_{M}^{(m)}}\right) i^{-\left(\sum_{\mu=1}^{m} p_{1}^{(\mu)}+\cdots+p_{M}^{(\mu)}+\left|s^{\mu}\right|\right)} \\
& \left.\times \sum_{j=0}^{\infty} \frac{(i \lambda)^{j+\sum_{\mu=1}^{m} p_{1}^{(\mu)}+\cdots+p_{M}^{(\mu)}+\left|s^{\mu}\right|}}{\left(j+\sum_{\mu=1}^{m} p_{1}^{(\mu)}+\cdots+p_{M}^{(\mu)}+\left|s^{\mu}\right|\right) !} \frac{\left(j+\sum_{\mu=1}^{m} p_{1}^{(\mu)}+\cdots+p_{M}^{(\mu)}+\left|s^{\mu}\right|\right) !}{j !} \xi^{j}\right)\left.\right|_{\xi=0} \\
& =\sum_{m=0}^{\infty} \frac{(i x)^{m}}{m !} \sum_{s^{1} \in \mathcal{M}} \cdots \sum_{s^{m} \in \mathcal{M}}\left(b_{q_{1}}^{\left(s_{1}^{1}\right)^{c}} \ldots b_{q_{M}}^{\left(s_{M}^{1}\right)^{c}}\right) \cdots\left(b_{q_{1}}^{\left(s_{1}^{m}\right)^{c}} \ldots b_{q_{M}}^{\left(s_{M}^{m}\right)^{c}}\right)(-1)^{\sum_{\mu=1}^{m}\left|s^{\mu}\right|} \\
& \times\left(\sum_{p_{1}^{(1)}=0, \ldots, p_{M}^{(1)}=0}^{\infty} \bar{b}_{q_{1}}^{p_{1}^{(1)}} \cdots \bar{b}_{q M}^{p_{M}^{(1)}}\right) \times \ldots \\
& \times\left(\sum_{p_{1}^{(m)}=0, \ldots, p_{M}^{(m)}=0}^{\infty} \bar{b}_{q_{1}}^{p_{1}^{(m)}} \cdots \bar{b}_{q_{M}}^{p_{M}^{(m)}}\right) \lambda^{\sum_{\mu=1}^{m} p_{1}^{(\mu)}+\cdots+p_{M}^{(\mu)}+\left|s^{\mu}\right|} \\
& =\sum_{m=0}^{\infty} \frac{(i x)^{m}}{m !}\left(B^{m}(\lambda)\right)=e^{i x B(\lambda)}
\end{aligned}
$$

for $|\lambda| \leq \min (\alpha, 1)$. 
Theorem 4.4 Let $\left(b_{i}\right)_{i=1}^{M}$ be a finite sequence of complex numbers such that $0<\left|b_{i}\right|<$ 1 for $i=1, \ldots, M$. Let $B(z)$ be the finite Blaschke product (32) and set

$$
Y_{n}(x, a):=\sum_{j=0}^{n} C_{j}(n, a) e^{i B(\varepsilon(1-2 j / n)) x}, \text { for } \varepsilon \in(0,1)
$$

Then we have

$$
\lim _{n \rightarrow \infty} Y_{n}(x, a)=e^{i B(\varepsilon a) x}
$$

for $a \in \mathbb{R}$ such that $\varepsilon|a|<1$.

Proof We define the operator $\mathcal{U}_{B}\left(x, \partial_{\xi}\right)$ as in (33). By Theorem 4.3 we obtain the relations

$$
e^{i x B(\varepsilon(1-2 j / n))}=\left.\mathcal{U}_{B}\left(x, \partial_{\xi}\right) e^{i \xi(\varepsilon(1-2 j / n))}\right|_{\xi=0} .
$$

and

$$
\left.\mathcal{U}_{B}\left(x, \partial_{\xi}\right) e^{i \varepsilon a \xi}\right|_{\xi=0}=e^{i x B(\varepsilon a)}
$$

Thus we have

$$
\begin{aligned}
\lim _{n \rightarrow \infty} Y_{n}(x, a) & =\lim _{n \rightarrow \infty} \sum_{j=0}^{n} C_{j}(n, a) e^{i x B(\varepsilon(1-2 j / n))} \\
& \left.\stackrel{(37)}{=} \lim _{n \rightarrow \infty} \sum_{j=0}^{n} C_{j}(n, a) \mathcal{U}_{B}\left(x, \partial_{\xi}\right) e^{i \xi(\varepsilon(1-2 j / n))}\right|_{\xi=0} \\
& =\left.\left.\lim _{n \rightarrow \infty} \mathcal{U}_{B}\left(x, \partial_{\xi}\right) \sum_{j=0}^{n} C_{j}(n, a) e^{i \xi(\varepsilon(1-2 j / n))}\right|_{\xi=0} \stackrel{\text { Theorem } \left.4.3 \text { (the continuity of } \mathcal{U}_{B}\left(x, \partial_{\xi}\right)\right)}{=} \mathcal{U}_{B}\left(x, \partial_{\xi}\right) e^{i \varepsilon a \xi}\right|_{\xi=0} \stackrel{(38)}{=} e^{i x B(\varepsilon a)}
\end{aligned}
$$

where we have assumed $|\varepsilon a|<1$.

We conclude this paper by observing that it is also possible to combine the relativistic sum of Blaschke products and superoscillations. These leads to more complicated computations because if we consider the Blaschke products

$$
B(z)=\prod_{n=1}^{N}\left(\frac{b_{n}-z}{1-\bar{b}_{n} z}\right), \quad C(z)=\prod_{m=1}^{M}\left(\frac{c_{m}-z}{1-\bar{c}_{m} z}\right)
$$

we have to deal with terms such as

$$
B(z) C(z)=\prod_{n=1}^{N} \prod_{m=1}^{M}\left(\frac{b_{n}-z}{1-\bar{b}_{n} z}\right)\left(\frac{c_{m}-z}{1-\bar{c}_{m} z}\right)
$$


in the expansion

$$
\frac{B+C}{1+B C}=(B+C) \sum_{\ell=0}^{\infty}(-1)^{\ell}(B C)^{\ell} .
$$

From these expressions we deduce suitable infinite order differential and the we have to study their continuity properties on the space of entire functions $\mathcal{A}_{1}$.

\section{Concluding remarks}

Recently in the paper [5] the authors have solved explicitly the following problem:

Problem 5.1 Let $h_{j}(n)$ be a given set of points in $[-1,1], j=0,1, \ldots, n$, for $n \in \mathbb{N}$ and let $a \in \mathbb{R}$ be such that $|a|>1$. Determine the coefficients $X_{j}(n)$ of the sequence

$$
f_{n}(x)=\sum_{j=0}^{n} X_{j}(n) e^{i h_{j}(n) x}, \quad x \in \mathbb{R}
$$

in such a way that

$$
f_{n}^{(p)}(0)=(i a)^{p}, \text { for } p=0,1, \ldots, n .
$$

Remark 5.2 The conditions $f_{n}^{(p)}(0)=(i a)^{p}$ mean that the functions $x \mapsto e^{i a x}$ and $f_{n}(x)$ have the same derivatives at the origin, for $p=0,1, \ldots, n$, so they have the same Taylor polynomial of order $n$.

Under the condition that the points $h_{j}(n)$ for $j=0, \ldots, n$, (often denoted by $h_{j}$ ) are distinct we obtain an explicit formula for the coefficients $X_{j}(n, a)$ given by

$$
X_{j}(n, a)=\prod_{k=0, k \neq j}^{n}\left(\frac{h_{k}(n)-a}{h_{k}(n)-h_{j}(n)}\right)
$$

so the superoscillating sequence $f_{n}(x)$, that solves Problem 5.1, takes the explicit form

$$
f_{n}(x)=\sum_{j=0}^{n} \prod_{k=0, k \neq j}^{n}\left(\frac{h_{k}(n)-a}{h_{k}(n)-h_{j}(n)}\right) e^{i h_{j}(n) x}, \quad x \in \mathbb{R} .
$$

Observe that, by construction, this function is band limited and it converges to $e^{\text {iax }}$ with arbitrary $|a|>1$, so it is superoscillating.

Different sequences $X_{j}(n)$ can be explicitly computed when we fix the configurations of the points $h_{j}(n)$. For example, with the configuration of points

$$
h_{j}(n)=1-2 j / n^{p} \text { for } j=0, \ldots, n, \quad n \in \mathbb{N} \text { and for fixed } p \in \mathbb{N},
$$


we obtain:

$$
f_{n}(x)=\sum_{j=0}^{n} \prod_{k=0, k \neq j}^{n} \frac{n^{p}}{2}\left(\frac{1-\frac{2}{n^{p}} k-a}{j-k}\right) e^{i\left(1-\frac{2}{n^{p}} j\right) x}, \quad x \in \mathbb{R} .
$$

It is worthwhile to mention that in antenna theory the phenomenon of superoscillations was discovered by G. Toraldo di Francia in [34]. The literature on superoscillations from the physics point of view is large, but since physical aspects are outside the scope of this paper we mention here only [22] and the recent paper Roadmap on superoscillations, see [21], in which some of the most important achievements in the applications of superoscillations are well explained by the leading experts in this field.

More information on the mathematical approach to superoscillations can be found in the introductory papers $[13,14,17,33]$.

Acknowledgements The authors would like to thank both the referees for carefully reading the manuscript and for their comments. The second author is partially supported by the PRIN project Direct and inverse problems for partial differential equations: theoretical aspects and applications.

Funding Open access funding provided by Politecnico di Milano within the CRUI-CARE Agreement.

\section{Declarations}

Conflict of interest The authors declare that they have no conflict of interest.

Open Access This article is licensed under a Creative Commons Attribution 4.0 International License, which permits use, sharing, adaptation, distribution and reproduction in any medium or format, as long as you give appropriate credit to the original author(s) and the source, provide a link to the Creative Commons licence, and indicate if changes were made. The images or other third party material in this article are included in the article's Creative Commons licence, unless indicated otherwise in a credit line to the material. If material is not included in the article's Creative Commons licence and your intended use is not permitted by statutory regulation or exceeds the permitted use, you will need to obtain permission directly from the copyright holder. To view a copy of this licence, visit http://creativecommons.org/licenses/by/4.0/.

\section{References}

1. Aharonov, Y., Albert, D., Vaidman, L.: How the result of a measurement of a component of the spin of a spin-1/2 particle can turn out to be 100. Phys. Rev. Lett. 60, 1351-1354 (1988)

2. Aharonov, Y., Behrndt, J., Colombo, F., Schlosser, P.: Green's function for the Schrödinger equation with a generalized point interaction and stability of superoscillations. J. Differential Equations 277, 153-190 (2021)

3. Aharonov, Y., Behrndt, J., Colombo, F., Schlosser, P.: Schrödinger evolution of superoscillations with $\delta$ - and $\delta^{\prime}$-potentials. Quantum Stud. Math. Found. 7(3), 293-305 (2020)

4. Aharonov, Y., Colombo, F., Nussinov, S., Sabadini, I., Struppa, D.C., Tollaksen, J.: Superoscillation phenomena in $S O$ (3). Proc. Royal Soc. A. 468, 3587-3600 (2012)

5. Y. Aharonov, F. Colombo, I. Sabadini, T. Shushi, D. C. Struppa, J. Tollaksen, A new method to generate superoscillating functions and supershifts, arXiv:2101.06416, to appear in Proceeding A, 2021

6. Aharonov, Y., Colombo, F., Sabadini, I., Struppa, D.C., Tollaksen, J.: Some mathematical properties of superoscillations. J. Phys. A 44, 365304 (2011)

7. Aharonov, Y., Colombo, F., Sabadini, I., Struppa, D.C., Tollaksen, J.: On the Cauchy problem for the Schrödinger equation with superoscillatory initial data. J. Math. Pures Appl. 99, 165-173 (2013)

8. Aharonov, Y., Colombo, F., Sabadini, I., Struppa, D.C., Tollaksen, J.: Superoscillating sequences as solutions of generalized Schrodinger equations. J. Math. Pures Appl. 103, 522-534 (2015) 
9. Aharonov, Y., Colombo, F., Sabadini, I., Struppa, D.C., Tollaksen, J.: How superoscillating tunneling waves can overcome the step potential. Ann. Phys. 414, 168088 (2020)

10. Aharonov, Y., Colombo, F., Sabadini, I., Struppa, D.C., Tollaksen, J.: Superoscillating sequences in several variables. J. Fourier Anal. Appl. 22, 751-767 (2016)

11. Aharonov, Y., Colombo, F., Sabadini, I., Struppa, D.C., Tollaksen, J.: The mathematics of superoscillations. Mem. Am. Math. Soc. 247(1174), 107 (2017)

12. Aharonov, Y., Colombo, F., Sabadini, I., Struppa, D.C., Tollaksen, J.: Evolution of superoscillations in the Klein-Gordon field. Milan J. Math. 88, 171-189 (2020)

13. Aharonov, Y., Colombo, F., Struppa, D.C., Tollaksen, J.: Schrödinger evolution of superoscillations under different potentials. Quan. Stud. Math. Found. 5, 485-504 (2018)

14. Aharonov, Y., Sabadini, I., Tollaksen, J., Yger, A.: Classes of superoscillating functions. Quant. Stud. Math. Found. 5, 439-454 (2018)

15. Aharonov, Y., Vaidman, L.: Properties of a quantum system during the time interval between two measurements. Phys. Rev. A 41, 11-20 (1990)

16. Alpay, D., Colombo, F., Sabadini, I.: Superoscillations and analytic extension in schur analysis. J. Fourier Anal. Appl. 27(2), 28 (2021)

17. Aoki, T., Colombo, F., Sabadini, I., Struppa, D.C.: Continuity of some operators arising in the theory of superoscillations. Quant. Stud. Math. Found. 5, 463-476 (2018)

18. Aoki, T., Colombo, F., Sabadini, I., Struppa, D.C.: Continuity theorems for a class of convolution operators and applications to superoscillations. Ann. Mat. Pura Appl. 197, 1533-1545 (2018)

19. Behrndt, J., Colombo, F., Schlosser, P.: Evolution of Aharonov-Berry superoscillations in Dirac $\delta$ potential. Quan. Stud. Math. Found. 6, 279-293 (2019)

20. Berenstein, C.A., Gay, R.: Complex Analysis and Special Topics in Harmonic Analysis. SpringerVerlag, New York (1995)

21. Berry, M., et al.: Roadmap on superoscillations. J. Opt. 21, 053002 (2019)

22. M. V. Berry, Faster than Fourier, in Quantum Coherence and Reality; in celebration of the 60th Birthday of Yakir Aharonov ed. J.S.Anandan and J. L. Safko, World Scientific, Singapore, (1994), pp. 55-65. ditors (Springer,New York), pp.327-336

23. Berry, M.V.: Representing superoscillations and narrow Gaussians with elementary functions. Milan J. Math. 84, 217-230 (2016)

24. Buniy, R., Colombo, F., Sabadini, I., Struppa, D.C.: Quantum harmonic oscillator with superoscillating initial datum. J. Math. Phys. 55, 113511 (2014)

25. Colombo, F., Gantner, J., Struppa, D.C.: Evolution by Schrödinger equation of Aharonov-Berry superoscillations in centrifugal potential. Proc. A 475(2225), 20180390 (2019)

26. Colombo, F., Gantner, J., Struppa, D.C.: Evolution of superoscillations for Schrödinger equation in a uniform magnetic field. J. Math. Phys. 58(9), 092103 (2017)

27. Colombo, F., Struppa, D.C., Yger, A.: Superoscillating sequences towards approximation in $\mathcal{S}$ or $\mathcal{S}^{\prime}$-type spaces and extrapolation. J. Fourier Anal. Appl. 25(1), 242-266 (2019)

28. F. Colombo, I. Sabadini, D.C. Struppa, A. Yger, Superoscillating sequences and supershifts for families of generalized functions, Preprint (2019)

29. Colombo, F., Sabadini, I., Struppa, D.C., Yger, A.: Gauss sums, superoscillations and the Talbot carpet. J. Math. Pures Appl. 147, 163-178 (2021)

30. Colombo, F., Sabadini, I., Struppa, D.C., Yger, A.: Superoscillating sequences and hyperfunctions. Publ. Res. Inst. Math. Sci. 55(4), 665-688 (2019)

31. F. Colombo, I. Sabadini, D.C. Struppa, A. Yger, Superoscillating sequences and supershifts for families of generalized functions, preprint 2019, arXiv:1912.01057

32. Colombo, F., Valente, G.: Evolution of superoscillations in the Dirac field. Found. Phys. 50(11), 13561375 (2020)

33. Kempf, A.: Four aspects of superoscillations. Quan. Stud. Math. Found. 5, 477-484 (2018)

34. Super-Gain Antennas and Optical Resolving Power: Toraldo di Francia, G. Nuovo Cimento Suppl. 9, 426-438 (1952)

35. L. Susskind, A. Friedman, Special relativity and classical field theory. The theoretical minimum, Basic Books, New York, 2017. pp. xx+425

Publisher's Note Springer Nature remains neutral with regard to jurisdictional claims in published maps and institutional affiliations. 